\title{
QPLIB: A Library of Quadratic Programming Instances
}

\author{
Fabio Furini Emiliano Traversi Pietro Belotti \\ Antonio Frangioni Ambros Gleixner Nick Gould Leo Liberti \\ Andrea Lodi Ruth Misener Hans Mittelmann Nick Sahinidis \\ Stefan Vigerske Angelika Wiegele
}

\begin{abstract}
This paper describes a new instance library for Quadratic Programming (QP), i.e., the family of continuous and (mixed)-integer optimization problems where the objective function and/or the constraints are quadratic. QP is a very diverse class of problems, comprising sub-classes ranging from trivial to undecidable. This diversity is reflected in the variety of QP solution methods, ranging from entirely combinatorial approaches to completely continuous algorithms, including many methods for which both aspects are fundamental. Selecting a set of instances of QP that is at the same time not overwhelmingly onerous but sufficiently challenging for the different, interested communities is therefore important. We propose a simple taxonomy for QP instances leading to a systematic problem selection mechanism. We then briefly survey the field of QP, giving an overview of theory, methods and solvers. Finally, we describe how the library was put together, and detail its final contents.
\end{abstract}

Keywords: Instance Library, Quadratic Programming

\section{Introduction}

Quadratic Programming (QP) problems - mathematical optimization problems for which the objective function [145], the constraints [146], or both are polynomial function of the variables of degree two - include a notably diverse set of different instances. This is not surprising, given the vast scope of practical applications of such problems, and of solution methods designed to solve them [68]. Depending on the formulation specifics, solving a QP may require primarily combinatorial techniques, ideas rooted in nonlinear optimization principles, or a mix of the two. In this sense, QP is a class of problems where collaboration between the communities interested in combinatorial and in nonlinear optimization is potentially fruitful.

However, this diversity also implies that QP means very different things to different researchers. This is illustrated by the fact that the class of problems that we simply 
refer to here as "QP" might more accurately be called Mixed-Integer QuadraticallyConstrained Quadratic Programming (MIQCQP) in the most general case. Therefore, it is perhaps not surprising that, unlike for "simpler" problems classes like Mixed-Integer Linear Programming [84], there has been no single library devoted to all different kinds of instances of QP. While several specialized libraries devoted to particular classes of QP are available, each of them is either focused on a particular application (a specific problem that can be modeled as a QP), or on QPs with specific structural properties that make them suitable for solution by some given class of algorithmic approaches. To the best of our knowledge, collecting a set of QP instances that is at the same time not overwhelmingly onerous but sufficiently challenging for the many different interested communities has not been attempted. This work constitutes a first step in this direction.

This paper reports our steps towards collecting what we consider to be a quality QP instance library, filtering a much larger set of currently available (or specifically provided) instances and proposing a manageable set that still contains a meaningful sample of possible QP types. A particularly thorny issue in this process was how to select instances that are "interesting". Our choice has been to take this to mean "challenging for a significant set of solution methods". Our filtering process has then been in part based on the idea that, if a significant fraction of the solvers that can solve a QP instance do so in a "short" time, then the instance is not challenging enough to be included in the library. Conversely, if very few (maybe one) of the solvers can solve it very efficiently by exploiting some specific structure, but most other approaches cannot, then the instance should be deemed "interesting". Putting this approach into practice requires a nontrivial number of technical steps and decisions that are detailed in the paper. We hope that our work can provide useful guidelines for other researchers interested in constructing benchmarks for mathematical optimization problems.

A consequence of our focus is that this paper is not concerned with the performance of the very diverse available set of QP solvers; we will not report any data comparing them. The only reason that solvers are used (and, therefore, described) in this context is to ensure that the library instances are nontrivial, at least for a significant fraction of the current solution methods. Providing guidance about which solvers are most suited to some specific class of QPs is entirely outside the scope of our work.

\subsection{Motivation}

Optimization problems with quadratic constraints and/or objective function (QP) have been the subject of a considerable amount of research for almost seventy years. Part of the rationale for this interest is that QPs are the "least-nonlinear nonlinear problems". Hence, in particular for the convex case, tools and techniques that have been honed during decades of research for Linear Programming (LP), typically with integrality constraints (MILP), can often be extended to the quadratic case more easily than would be required to tackle general (Mixed-Integer) Nonlinear Programming ((MI)NLP) problems. This has indeed happened over-and-over again with different algorithmic techniques, such as interior-point methods, active-set methods, e.g., the simplex method, enumeration methods, cut-generation techniques, reformulation techniques, and many others [26]. Similarly, nonconvex continuous QP is perhaps the "simplest" class of problems that require features such as spatial enumeration techniques for their solution. Hence, QPs are both a natural basis for developing general techniques for nonconvex NLP, and a very 
specific class enabling the development of specialized approaches [27, 44].

In addition, QP, with continuous or integer variables, is arguably a considerably more expressive class than (MI)LP. Quadratic expressions are found, either naturally or after appropriate reformulations, in very many optimization problems [85]. Table 1 provides a non-exhaustive collection of applications that lead to formulations with quadratic constraints, quadratic objective function, or both. In general, any continuous function can be approximated with arbitrary accuracy (over a compact set) by a polynomial of arbitrary degree. In turn, every polynomial can be broken down to a system of quadratic expressions. Hence, QP is, in some sense, roughly as expressive as MINLP. This is, in principle, true for MILP as well, but at the cost of much larger and much more complicated formulations. Hence, for many applications QP may represent the "sweet spot" between the effectiveness, but lower expressive power, of MILP and the higher expressive power, but much higher computational cost, of MINLP.

Table 1: Application Domains of QP

\begin{tabular}{|c|c|c|}
\hline Problem & Discrete & Contributions \\
\hline \multicolumn{3}{|c|}{ Fundamental problems that can be formulated as MIQP } \\
\hline Quadratic assignment problem ${ }^{\ddagger}$ & $\checkmark$ & {$[8,100]$} \\
\hline Max-cut & $\checkmark$ & {$[89,120]$} \\
\hline Maximum clique ${ }^{\ddagger}$ & $\checkmark$ & {$[22]$} \\
\hline \multicolumn{3}{|c|}{ Computational chemistry \& Molecular biology } \\
\hline Zeolites & & {$[72]$} \\
\hline \multicolumn{3}{|l|}{ Computational geometry } \\
\hline Layout design & $\checkmark$ & {$[7,30,39]$} \\
\hline Maximizing polygon dimensions & & {$[9-13]$} \\
\hline Packing circles ${ }^{\ddagger}$ & $\checkmark$ & {$[51,57,76,129]$} \\
\hline Nesting polygons & & {$[81,119]$} \\
\hline Cutting ellipses & & {$[82]$} \\
\hline \multicolumn{3}{|l|}{ Finance } \\
\hline Portfolio optimization & $\checkmark$ & $\begin{array}{l}{[37,51,54-} \\
56,80,98,101,113,122]\end{array}$ \\
\hline \multicolumn{3}{|l|}{ Process networks } \\
\hline Crude oil scheduling & $\checkmark$ & {$[93-95,106,107]$} \\
\hline Data reconciliation & $\checkmark$ & {$[124]$} \\
\hline Multi-commodity flow & $\checkmark$ & {$[130]$} \\
\hline Quadratic network design & $\checkmark$ & {$[51,57]$} \\
\hline Multi-period blending & $\checkmark$ & {$[87,88]$} \\
\hline Natural gas networks & $\checkmark$ & {$[74,96,97]$} \\
\hline
\end{tabular}

${ }^{\ddagger}$ Applications with many manuscripts cite reviews and recent works 
Table 1 (Application Domains of QP) continued

\begin{tabular}{|c|c|c|}
\hline Problem & Discrete & Contributions \\
\hline Pooling ${ }^{\ddagger}$ & $\checkmark$ & $\begin{array}{l}{[4,31,36,47,102,103} \\
112,114,125]\end{array}$ \\
\hline Open-pit mine scheduling & $\checkmark$ & {$[20]$} \\
\hline Reverse osmosis & $\checkmark$ & {$[126]$} \\
\hline Supply chain & $\checkmark$ & [111] \\
\hline Water networks ${ }^{\ddagger}$ & $\checkmark$ & $\begin{array}{l}{[3,14,24,33,58,64,79,} \\
83,118,136]\end{array}$ \\
\hline
\end{tabular}

\section{Robotics}

Traveling salesman problem with neighborhoods

$\checkmark \quad[59]$

\begin{tabular}{lcl}
\hline Telecommunications & & \\
Delay-constrained routing & $\checkmark$ & {$[52,53]$} \\
\hline Energy & & \\
Unit-commitment & $\checkmark$ & {$[51,54,56,131]$} \\
\hline Data confidentiality & & \\
Controlled Tabular Adjustment & $\checkmark$ & {$[32]$} \\
\hline
\end{tabular}

\section{Trust-region methods}

Trust-region subproblem

$[2,46,70,71,73,121]$

\section{PDE-constrained optimization}

Optimal control problem

$[115,127,128]$

${ }^{\ddagger}$ Applications with many manuscripts cite reviews and recent works.

The structure of this paper is as follows. In $\S 2$ we review the basic notion of QP. In particular, $\S 2.1$ sets out the notation, $\S 2.2$ proposes a new QP taxonomy that helps discuss the (very) different QP classes, and $\S 2.3$ very briefly reviews the QP solution methods and the solvers we have employed. Next, $\S 3$ describes the process used to obtain the library and its results. Some conclusions are drawn in $\S 4$, after which Appendix A provides a complete description of all the instances of the library, while Appendix B describes a simple (QPLIB) file format that encodes all of our examples.

While no performance issues of solvers for QP problems are considered in this paper, we refer to the comprehensive benchmark site http://plato.asu.edu/bench.html.Of the result on this site, three deal exclusively with QP problems, namely the (1) large SOCP, (2) MISOCP, and the (3) MIQ(C)P benchmarks, while three others contain partial results for such problems, namely those for (4) parallel barrier solvers on large LP/QP problems, (5) AMPL-NLP and (6) MINLP. Benchmarks (1, 2 \& 4) contain only convex instances, while the others include nonconvex ones. Global optima are obtained by several of the solvers in benchmarks ( $3 \& 5$ ), while all solvers in the latest addition (6) compute global optima. Benchmark (6) is based on MINLPLib 2 [139], a collection of currently 1527 instances. In order to give a first representative impression of solver performance, 
care was taken there to reduce the number of instances and allow all solvers to finish in a reasonable time. More than half of the selected instances are QP or QCP. For details we refer to http://plato.asu.edu/ftp/minlp.html.

\section{Quadratic Programming in a Nutshell}

\section{$2.1 \quad$ Notation}

In mathematical optimization, a Quadratic Program (QP) is an optimization problem in which either the objective function, or some of the constraints, or both, are quadratic functions. More specifically, the problem has the form

$$
\begin{aligned}
& \min \text { or max } \frac{1}{2} x^{\top} Q^{0} x+b^{0} x+q^{0} \\
& \text { such that } c_{l}^{i} \leq \frac{1}{2} x^{\top} Q^{i} x+b^{i} x \leq c_{u}^{i} \quad i \in \mathcal{M} \text {, } \\
& l_{j} \leq x_{j} \leq u_{j} \quad j \in \mathcal{N}, \\
& \text { and } x_{j} \in \mathbb{Z} \quad j \in \mathcal{Z} \text {, }
\end{aligned}
$$

where

- $\mathcal{N}=\{1, \ldots, n\}$ is the set of (indices) of variables, and $\mathcal{M}=\{1, \ldots, m\}$ is the set of (indices) of constraints;

- $x=\left[x_{j}\right]_{j=1}^{n}$ is a finite vector of real variables;

- $Q^{i}$ for $i \in\{0\} \cup \mathcal{M}$ are symmetric $n \times n$ real (Hessian) matrices: since one is only interested in the value of quadratic forms of the type $x^{\top} Q^{i} x$, symmetry can be assumed without loss of generality by just replacing off diagonal pairs $Q_{h k}^{i}$ and $Q_{k h}^{i}$ with their average $\left(Q_{h k}^{i}+Q_{k h}^{i}\right) / 2$;

- $b^{i}, c_{u}^{i}, c_{l}^{i}$ for $i \in\{0\} \cup \mathcal{M}$, and $q^{0}$ are, respectively, real $n$-vectors and real constants;

- $-\infty \leq l_{j} \leq u_{j} \leq \infty$ are the (extended) real lower and upper bounds on each variable $x_{j}$ for $j \in \mathcal{N}$;

- $\mathcal{M}=\mathcal{Q} \cup \mathcal{L}$ where $Q^{i}=0$ for all $i \in \mathcal{L}$ (i.e., these are the linear constraints, as opposed to the truly quadratic ones); and

- the variables in $\mathcal{Z} \subseteq \mathcal{M}$ are restricted to only attain integer values.

Due to the quadratic constraints and the integrality requirements on the variables, this class is often referred to as Mixed-Integer Quadratically Constraint Quadratic Program (MIQCQP). It will be sometimes useful to refer to the (sub)set $\mathcal{B}=\left\{j \in \mathcal{Z}: l_{j}=0, u_{j}=\right.$ $1\} \subseteq \mathcal{Z}$ of the binary variables, and to $\mathcal{R}=\mathcal{N} \backslash \mathcal{Z}$ as the set of continuous variables. Similarly, it will be sometimes useful to distinguish the (sub)set $\mathcal{X}=\left\{j: l_{j}>-\infty \vee u_{j}<\right.$ $\infty\}$ of the box-constrained variables from $\mathcal{U}=\mathcal{N} \backslash \mathcal{X}$ of the unconstrained ones (in the sense that finite bounds are not explicitly provided in the problem data, although bounds may be implied by the other constraints).

The relative flexibility offered by quadratic functions, as opposed, e.g., to linear ones, allows several reformulation techniques to be applicable to this family of problems in order 
to emphasize different properties of the various components. Some of these reformulation techniques will be commented later on; here we remark that, for instance, integrality requirements, in particular in the form of binary variables could always be "hidden" by introducing (nonconvex) quadratic constraints utilizing the celebrated relationship $x_{j} \in\{0,1\} \Longleftrightarrow x_{j}^{2}=x_{j}$. Therefore, when discussing these problems, some effort has to be made to distinguish between features that come from the original model, and those that can be introduced by reformulation techniques in order to extract (and algorithmically exploit) specific properties.

\subsection{Classification}

Despite the apparent simplicity of the $§ 2.1$ definition, Quadratic Programming instances can be of several rather different "types" in practice, depending on fine details of the data. In particular, many algorithmic approaches can only be applied to QP when the problem data has specific properties. A taxonomy of QP instances should thus strive to identify a set of properties that an instance should have in order to apply the most relevant computational methods. However, the sheer number of different existing approaches, and the fact that new ones are frequently proposed, makes it hard to provide a taxonomy that is both simple and covers all possible special cases. This is why, in this paper, we propose an approach that aims at finding a good balance between simplicity and coverage of the main families of computational methods.

\subsubsection{Taxonomy}

Our taxonomy is based on a three-fields code of the form "OVC", where $O$ indicates the type of objective function considered, $V$ records the types of variables, and $C$ designates the types of constraints imposed on the variables. The fields can be given the following values:

- objective function: ( $L$ )inear, $(D)$ iagonal convex (if minimization) or concave (if maximization) quadratic, $(C)$ onvex (if minimization) or $(C$ )oncave (if maximization) quadratic, $(Q)$ uadratic (all other cases);

- variables: $(C)$ ontinuous only, $(B)$ inary only, $(M)$ ixed binary and continuous, $(I)$ nteger (including binary) only, $(G)$ eneral (all other cases);

- constraints: $(N)$ one, $(B)$ ox, $(L)$ inear, $(D)$ iagonal convex quadratic, $(C)$ onvex quadratic, nonconvex $(Q)$ uadratic. Note that (positive or negative) definiteness of $Q^{i}$ is a sufficient, but not in general necessary, condition for convexity. As detailed in $\S 3.3$, in our taxonomy we mark the constraints " $C$ " based on the sufficient condition alone, the rationale of this choice being discussed in $§ 2.2 .2$. Quadratic constraints with both finite bounds cannot ever be convex (unless $Q^{i}=0$, i.e., they are not "truly" quadratic constraints).

The ordering in the preceding lists is relevant; in general, problems become "harder" when going from left to right. More specifically, for the $O$ and $C$ fields the order is that of strict containment between problem classes: for instance, linear objective functions are strictly a special case of diagonal convex quadratic ones (by allowing the diagonal elements all to be zero), the latter are a strict subset of general convex quadratic objectives (by 
allowing the off-diagonal elements all to be zero), and these are strictly subsets of general nonconvex quadratic ones (since these permit any symmetric Hessian including positive semidefinite ones). The only field for which the containment relationship is not a total order is $V$, for which only the partial orderings

$$
C \subset M \subset G, \quad B \subset M \subset G, \quad \text { and } \quad B \subset I \subset G
$$

hold. The following discussion repeatedly exploits this ordering by assuming that, unless otherwise mentioned, when a method can be applied to a given problem, it can also be applied to all simpler problems where the value of each field is arbitrarily replaced with a value denoting a less-general class.

The wildcard "*" will be used below to indicate any possible choice, and lists of the form " $\{X, Y, Z\}$ " will indicate that the value of the given field can freely attain any of the specified values.

\subsubsection{Examples and Reformulations}

We now give a general discussion about the different problem classes that our proposed taxonomy defines. For simplicity, this section assumes minimization problems. Some problem classes are actually "too simple" to make sense in our context. For instance, $D^{*} B$ problems have only diagonal quadratic (hence separable) objective function and bound constraints; as such, they read

$$
\min \left\{\sum_{j \in \mathcal{N}}\left(\frac{1}{2} Q_{j}^{0} x_{j}^{2}+b_{j}^{0} x_{j}\right): l_{j} \leq x_{j} \leq u_{j} \quad j \in \mathcal{N}, x_{j} \in \mathbb{Z} \quad j \in \mathcal{Z}\right\} .
$$

Hence, their solution only requires the independent minimization of a convex quadratic univariate function in each single variable $x_{j}$ over a box constraint and possibly integrality requirements, which can be attained trivially in $O(1)$ operations (per variable) by closedform formulæ, projection and rounding arguments. A fortiori, the even simpler cases $L^{*} B, D^{*} N$ and $L^{*} N$ (the latter unbounded unless $b^{0}=0$ ) will not be discussed here. Similarly, $C C N$ are immediately solved by linear algebra techniques, and therefore are of no interest in this context. At the other end of the spectrum, in general QP is a hard problem. Actually, $L I Q$ - linear objective function and quadratic constraints in integer variables with no finite bounds, i.e.

$$
\min \left\{b^{0} x: \frac{1}{2} x^{\top} Q^{i} x+b^{i} x \leq c^{i} \quad i \in \mathcal{M}, x_{j} \in \mathbb{Z} \quad j \in \mathcal{N}\right\}
$$

is not only $\mathcal{N} \mathcal{P}$-hard, but undecidable [78]. Hence so are the "harder" $\{C, Q\} I Q$.

It is important to note that the relationships between the different classes can be somehow blurred because reformulation techniques may allow one to move an instance from one class to another. We already mentioned that $x^{2}=x \Longleftrightarrow x \in\{0,1\}$, and in general ${ }^{*} M *$ - instances with only binary and continuous variables - can be recast as ${ }^{*} C Q$; here nonconvex quadratic constraints take the place of binary variables. More generally, this is also true for ${ }^{*} G^{*}$ as long as $\mathcal{U}=\emptyset$, as bounded general integer variables can be represented by binary ones. Hence, the nonconvexity due to binary variables can always be expressed by means of (nonconvex) quadratic constraints. The converse is also true: when only binary variables are present, all quadratic constraints can be converted into convex ones $[17,18]$. 
Another relevant reformulation trick concerns the fact that, as soon as quadratic constraints are allowed, then there is no loss of generality in assuming a linear objective function. Indeed, any $Q^{* *}\left(C^{*} C\right)$ problem can always be rewritten as

$$
\begin{aligned}
& \min x^{0} \\
& -\infty \leq \frac{1}{2} x^{\top} Q^{0} x+b^{0} x \leq x^{0} \\
& c_{l}^{i} \leq \frac{1}{2} x^{\top} Q^{i} x+b^{i} x \leq c_{u}^{i} \quad i \in \mathcal{M} \\
& l_{j} \leq x_{j} \leq u_{j} \quad j \in \mathcal{N} \\
& x_{j} \in \mathbb{Z} \quad j \in \mathcal{Z}
\end{aligned}
$$

i.e., a $L^{*} Q\left(L^{*} C\right)$ problem. Hence, it is clear that quadratic constraints are, in a welldefined sense, the most general situation (cf. also the result above about hardness of $L I Q)$.

When a $Q^{i}$ is positive semidefinite (PSD), i.e., the corresponding constraint/objective function is convex, general Hessians are in fact equivalent to diagonal ones. In particular, since every PSD matrix can be factorized as $Q^{i}=L^{i}\left(L^{i}\right)^{\top}$, e.g. by the (incomplete) Cholesky factorization, the term $\frac{1}{2} x^{\top} Q^{i} x \equiv \frac{1}{2} \sum_{j \in \mathcal{N}} z_{j}^{i 2}$ where $z^{i \top}=x^{\top} L^{i}$. Hence, one might maintain that $\mathrm{D}^{* *}$ problems need not be distinguished from $\mathrm{C}^{* *}$ ones. However in reality, this is only true for "complicated" constraints but not for "simple" ones, because the above reformulation technique introduces additional linear constraints, $L^{i \top} x-z^{i}=0$. Indeed, while $C^{*} L$ (and, a fortiori, $\left.C^{*}\{C, Q\}\right)$ can always be brought to $D^{*} L\left(D^{*}\{C, Q\}\right)$, using the above technique $C^{*} B$ becomes $D^{*} L$, which may be significantly different from $D^{*} B$. In practice, a diagonal convex objective function under linear constraints is found in many applications (e.g., $[51,54,56,57]$ ), so that it still makes sense to distinguish the $D^{*} L$ case where the objective function is "naturally" separable from that where separability is artificially introduced.

Furthermore, as previously remarked, a not (positive or negative) definite $Q^{i}$ does not necessarily correspond to a nonconvex feasible region. For instance, it is well-known that Second-Order Cone Programs have convex feasible regions; when represented in terms of quadratic constraints, however, they correspond to $Q^{i}$ with one negative eigenvalue. In our taxonomy we still consider the corresponding instances as ${ }^{* *} Q$ ones, with no attempt to detect the different special structures that actually correspond to convex feasible regions. Although this may lead to classify as "potentially nonconvex" some instances that are in fact convex, our choice is justified by the fact that not all QP solvers are capable of detecting and exploiting these structures, which means that the instance can actually be treated as a nonconvex one even if it is not.

One of the nontrivial choices in our library is that we made no effort to reformulate the instances, and inserted them in the library in the very same form as they have been provided to us by the original contributors. The rationale of this choice is that reformulation techniques, like the ones discussed here and others, are typically motivated by the fact that they make the instance easier to solve for one specific class of solvers. This being a bias that we do not want to add we have chosen to keep the instances in their "natural" form, this being the one in which the original contributor initially wrote them. 


\subsubsection{QP Classes}

The proposed taxonomy can then be used to describe the main classes of QP according to the type of algorithms that can be applied for their solution:

- Linear Programs LCL and Mixed-Integer Linear Programs LGL have been subject of an enormous amount of research and have their well-established instance libraries [84], so they will not be explicitly addressed here.

- Convex Continuous Quadratic Programs CCC can be solved in polynomial time by Interior-Point techniques [147]; the simpler $C C L$ can also be solved by means of "simplex-like" techniques, usually referred to as active-set methods [40]. Actually, a slightly larger class of problems can be solved with Interior-Point methods: those that can be represented by Second-Order Cone Programs. When written as QPs the corresponding $Q^{i}$ may not be positive semidefinite, but nonetheless such problems can be efficiently solved. Of course, just as for $L C L$, these problems may still require considerable computational effort when the size of the instance grows. In this sense, like in the linear case, there is a significant distinction between solvers that need all the data of QP to work, and those that are "matrix-free", i.e., only require the application of simple operations (typically, matrix-vector products) with the problem data. When building our instance library we never exploited such characteristics, since they are not amenable to standard modeling tools, but this may be relevant for the solution of very-large-scale $C I C$.

- Nonconvex Continuous Quadratic Programs $Q C Q$ are generally $\mathcal{N} \mathcal{P}$-hard, even if the constraints are very specific $(Q C B)$ and only a single eigenvalue of $Q^{0}$ is negative [75]. They therefore require enumerative techniques, such as spatial Branch-and-Bound $[15,50]$, to be solved to optimality. Of course, local approaches are available that are able to efficiently provide saddle points (hopefully, local optima) of the $C Q C$, but providing global guarantees about the quality of the obtained solutions is challenging. In our library we have specifically focused on exact solution of the instances.

- Convex Integer Quadratic Programs CGC are, in general, $\mathcal{N P}$-hard, and therefore require enumerative techniques to be solved. However, convexity of the objective function and constraints implies that efficient techniques (see $C C C$ ) can be used at least to solve continuous relaxations. The general view is that $C G C$ are not, all other things being equal, substantially more difficult than $L G L$ to solve, especially if the objective function and/or the constraints have specific properties (e.g., $D G L$, $C G L)$. Often, integer variables are in fact binary ones, so several $C G C$ models are $C\{B, M\} C$ ones. In practice, binary variables are considered to lead to somewhat easier problems than general integer ones (cf. the cited result about hardness of unbounded integer quadratic programs) and several algorithmic techniques have been specifically developed for this special case. However, the general approaches for $C B C$ are basically the same as for $C G C$, so there is seldom the need to distinguish between the two classes as far as solvability is concerned, although matters can be different regarding actual solution cost. Programs with only binary variables $(C B C)$ can be easier than mixed-binary or integer ones $(C\{M, I\} C)$ because some techniques are specifically known for the binary-only case, cf. the next point [17]. 
Absence of continuous variables, even in the presence of integer ones $(C I C)$, can also lead to specific techniques [18].

- Nonconvex Binary Quadratic Programs $Q B\{B, N, L\}$ are $\mathcal{N} \mathcal{P}$-hard. However, the special nature of binary variables combined with quadratic forms allows for quite specific techniques to be developed, one of which is the reformulation of the problem as a $L B L$. Also, many well-known combinatorial problems can be naturally reformulated as problems of this class, and therefore a considerable number of results have been obtained by exploiting specific properties of the set of constraints [100, 120].

- Nonconvex Integer Quadratic Programs $Q G Q$ is the most general, and therefore is the most difficult, class. Due to the lack of convexity even when integrality requirements are removed, solution methods must typically combine several algorithmic ideas, such as enumeration (distinguishing the role of integral variables from that of continuous ones involved in nonconvex terms) and techniques that allow the efficient computation of bounds (e.g., outer approximation, semidefinite programming relaxation, ...). As in the convex case, $Q B Q, Q M Q$, and $Q I Q$ can benefit from more specific properties of the variables [25, 38].

This description is deliberately coarse; each of these classes can be subdivided into several others on the grounds of more detailed information about structures present in their constraints/objective function. These can have a significant algorithmic impact, and therefore can be of interest to researchers. Common structures are, e.g., network flows $[51,52,130]$ or knapsack-type linear constraints [51, 57], and semi-continuous variables $[52,53,57]$, or the fact that a nonconvex quadratic objective function/constraint can be reformulated as a second-order cone (hence, convex) one $[53,56,57]$. It would be very hard to collect a comprehensive list of all types of structures that might be of interest to any individual researcher, since these are as varied as the different possible approaches for specialized sub-classes of QP. For this reason we do not attempt such a more refined classification, and limit ourselves to the coarser one described in this section.

\subsection{Solution Methods and Solvers}

This section provides a quick overview of existing solution methods for QP, restricting ourselves to these implemented by the specific solvers considered in this paper (see §2.3.1). For each approach, we briefly describe the formulation they address according to the $\S 2.2$ classification. Many solvers implement more than one algorithm, which the user can choose at runtime. Moreover, algorithms are typically implemented in different ways within different solvers, so that the same conceptual algorithm can sometimes yield different results or performance measures on the same instances.

Solution methods for QP can be broadly organized in four categories [110]: incomplete, asymptotically complete, complete, and rigorous.

- Incomplete methods are only able to identify solutions, often locally optimal according to a suitable notion, and may even fail to find one even when one exists; in particular, they are typically unable to determine that an instance has no solution.

- Asymptotically complete methods can find a globally optimal solution with probability one in infinite time, but they cannot prove that a given instance is infeasible (see $\$ 2.3 .3$ below). 


\begin{tabular}{|c|c|c|c|c|c|c|c|}
\hline & & CGL & QGL & CGC & QGQ & $\mathrm{CCC}$ & QCQ \\
\hline ALPHAECP & {$[143,144]$} & $\mathrm{C}$ & I & $\mathrm{C}$ & I & $\mathrm{C}$ & I \\
\hline ANTIGONE & {$[104,105]$} & $\mathrm{C}$ & $\mathrm{C}$ & $\mathrm{C}$ & $\mathrm{C}$ & $\mathrm{C}$ & $\mathrm{C}$ \\
\hline BARON & [133-135] & $\mathrm{C}$ & $\mathrm{C}$ & $\mathrm{C}$ & $\mathrm{C}$ & $\mathrm{C}$ & $\mathrm{C}$ \\
\hline BONMIN & {$[23]$} & $\mathrm{C}$ & I & $\mathrm{C}$ & I & $\mathrm{C}$ & I \\
\hline CONOPT & {$[41,42]$} & & & & & $\mathrm{C}$ & I \\
\hline Couenne & [15] & $\mathrm{C}$ & $\mathrm{C}$ & $\mathrm{C}$ & $\mathrm{C}$ & $\mathrm{C}$ & $\mathrm{C}$ \\
\hline Cplex & {$[19,77]$} & $\mathrm{C}$ & $\mathrm{C}$ & $\mathrm{C}$ & & $\mathrm{C}$ & \\
\hline DICOPT & {$[45,86,141]$} & $\mathrm{C}$ & I & $\mathrm{C}$ & I & $\mathrm{C}$ & I \\
\hline Gurobi & {$[123]$} & $\mathrm{C}$ & & $\mathrm{C}$ & & $\mathrm{C}$ & \\
\hline IPOPT & {$[142]$} & & & & & $\mathrm{C}$ & I \\
\hline KnItRo & {$[29]$} & $\mathrm{C}$ & I & $\mathrm{C}$ & I & $\mathrm{C}$ & A \\
\hline LiNDO API & [99] & $\mathrm{C}$ & $\mathrm{C}$ & $\mathrm{C}$ & $\mathrm{C}$ & $\mathrm{C}$ & $\mathrm{C}$ \\
\hline LGO & {$[116,117]$} & & & & & $\mathrm{A}$ & $\mathrm{A}$ \\
\hline MINOS & {$[108,109]$} & & & & & $\mathrm{C}$ & I \\
\hline MOSEK & {$[5,6]$} & $\mathrm{C}$ & & $\mathrm{C}$ & & $\mathrm{C}$ & \\
\hline MSNLP & {$[91,137]$} & & & & & $\mathrm{C}$ & A \\
\hline OQNLP & {$[91,137]$} & A & A & $\mathrm{A}$ & A & $\mathrm{C}$ & A \\
\hline SBB & [43] & $\mathrm{C}$ & I & $\mathrm{C}$ & I & $\mathrm{C}$ & I \\
\hline SCIP & {$[1,140]$} & $\mathrm{C}$ & $\mathrm{C}$ & $\mathrm{C}$ & $\mathrm{C}$ & $\mathrm{C}$ & $\mathrm{C}$ \\
\hline SNOPT & {$[61,62]$} & & & & & C & I \\
\hline XPRESS-OPTIMIZER & [48] & $\mathrm{C}$ & & $\mathrm{C}$ & & $\mathrm{C}$ & \\
\hline
\end{tabular}

Table 2: Families of QP problems that can be tackled by each solver

- Complete methods find an approximate globally optimal solution within a prescribed optimality tolerance within finite time, or prove that none such exists (but see $\S 2.3 .4$ below); they are often referred to as exact methods in the computational optimization community.

- Rigorous methods find globally optimal solutions within given tolerances even in the presence of rounding errors, except for "near-degenerate cases". Since none of the solvers we are using can be classified as rigorous, we limit ourselves to declaring solvers complete.

We refer the interested reader to [16] and [92] for further details on the solution methods.

\subsubsection{Solvers}

When compiling QPLIB, we have worked with the QP solvers in the GAMS distribution ${ }^{1}$. Table 2 provides a list of these solvers, together with a classification of their algorithm, and references. For more details on the solvers, we refer to the given references, solver manuals, and the survey [28]. In the table, we mark a pair (solver, problem) with "I" if the solver accepts the problem as input but it is an incomplete solver for the problem, with "A" if it is asymptotically complete, with "C" if it is complete, and leave it blank if the solver won't accept the provided problem. When a solver implements several algorithms, we have chosen, for each problem class, the algorithm that potentially provides the "strongest" results ("C" > "A" > "I" > blank).

\subsubsection{Incomplete Methods}

Incomplete methods are usually realized as local search algorithms, asymptotically complete methods are usually realized by meta-heuristic methods such as multi-start or simulated

\footnotetext{
${ }^{1}$ https: //www.gams.com
} 
annealing, and complete methods for $\mathcal{N} \mathcal{P}$-hard problems such as QP are typically realized as implicit exhaustive exploration algorithms. However, these three categories may exhibit some overlap. For example, any deterministic method for solving $Q C Q$ locally is incomplete in general, but becomes complete for $C C C$, since any local optimum of a convex $\mathrm{QP}$ is also global. Therefore, when we state that a given algorithm is incomplete or (asymptotically) complete we mean that it is so the largest problem class that the solver naturally targets, although it may be complete on specific sub-classes. For example, interior point algorithms naturally target NLPs and are incomplete on NLPs, and therefore on $Q C Q$, but become complete for $C C C$. In general, all complete methods for a problem class $P$ must be complete for any problem class $Q \subseteq P$, while a complete method for $P$ might be incomplete for a class $R \supset P$.

The Table 2 solvers which implement incomplete methods for NLPs (a problem class containing $Q C Q$ ) are CONOPT, IPOPT, MINOS, SNOPT, and KNITRO. Note that all these solvers tackle the more general class of NLP, while we use them only for the considerably more restricted QP class. Aside from solvers provided by GAMS, there are a number of other, specialized, incomplete QP solvers, such as CQP [65], DQP [67] and OOQP [60] for convex problems, and BQPD [49], QPA [69] and QPB [34], QPC [66], SQIC [63] for nonconvex ones.

\subsubsection{Asymptotically Complete Methods}

An asymptotically complete method reaches a global minimum with certainty or at least with probability one if allowed to run indefinitely long, but has no means to know when a global minimizer has been found (see [110]). Most often, these methods are metaheuristics, involving an element of random choice, which exploit a given (heuristic) local search procedure.

The solvers in Table 2 which implement asymptotically complete methods are OQNLP and KNITRO (which apply to $Q G Q$ ) as well as MsNLP and certain sub-solvers of LGO (which apply to $Q C Q$ ).

\subsubsection{Complete Methods}

Complete methods are often referred to as exact in a large part of the mathematical optimization community. This term has to be used with care, as it implicitly makes assumptions on the underlying computational model that may not be acceptable in all cases. For example, the decision version of $Q C L$ is known to be in the complexity class $\mathcal{N} \mathcal{P}$ [138], whereas the same is not known about $L C Q$, even with zero objective. On the other hand, there exists a method for deciding feasibility of systems of polynomial equations and inequalities [132], including the solution of $L C Q$ with zero objective function.

To explain this apparent contradiction, we remark that the two statements refer to different computational models: the former is based on the Turing Machine (TM), whereas the latter is based on a computational model that allows operations on real numbers, e.g. the Real RAM (RRAM) machine [21]. Due to the potentially infinite nature of exact real arithmetic computations, exact computations on the RRAM necessarily end up being approximate on the TM. Analogously, a complete method may reasonably be called "exact" on a RRAM; however, the computers we use in practice are more akin to TMs than RRAMs, and therefore calling exact a solver that employs floating point computations 
is, technically speaking, stretching the meaning of the word. However, because the term is well understood in the computational optimization community, in the following we shall loosen the distinction between complete and exact methods, with either properties intended to mean "complete" in the sense of [110].

Nearly all of the complete solvers in Table 2 that address $\mathcal{N} \mathcal{P}$-hard problems (i.e. those in $Q G Q \backslash C C C$ ) are based on Branch-and-Bound (BB) [90]. When the BB algorithm is allowed to branch on coordinate directions corresponding to continuous variables, it is called spatial $\mathrm{BB}(\mathrm{sBB})[15,35]$. BB algorithms require exponential time in the worst case, and their exponential behavior unfortunately often shows up in practice. They can also be used heuristically (forsaking their completeness guarantee) in a number of ways, e.g. by terminating them early. The following solvers from Table 2 implement complete BB algorithms for $Q G Q$ or some subclasses:

- Antigone, BARon, Couenne, Lindo API, and SCIP for $Q G Q$;

- Cplex for $Q G L$ and $C G C$;

- Gurobi and Xpress-Optimizer for $Q B C$;

- Bonmin, Gurobi, Knitro, MOSEK, SBB, and Xpress-Optimizer for $C G C$.

We remark that the solvers BONMIN, KNITRO, and SBB from the latter category can be used as incomplete solvers for $Q G Q$. We also note that LGO implements an incomplete $\mathrm{BB}$ algorithm for $Q C Q$ by using bounds obtained from sampling.

Cutting plane approaches construct and iteratively improve a MILP $(L I L)$ relaxation of the problem $[45,144]$. The cutting planes for the MILP are generated by linearization (first-order Taylor approximation) of the nonlinearities. If the latter are convex, the MILP provides a valid lower bound for the problem. Additionally, incomplete methods can be used to provide local solutions. Therefore, these methods are complete on $C G C$ if a complete method is used to solve the MILP. The latter is typically based on BB, which is therefore a crucial technique also for this class of approaches. Solvers in Table 2 that implement complete cutting plane methods for $C G C$ are ALPHAECP, BONMIN (in the algorithmic mode B-OA), and DICOPT.

\section{Library Construction}

This section presents all the steps we performed to build the new instance library. In $\S 3.1$, we describe the set of gathered instances, and in $\S 3.2$ we present the features used to classify the instances. We describe the selection process used to filter the instances, and graphically present the main features of the selected instances in $\S 3.3$, while in $\S 3.4$ we provide information on how to access the test collection.

\subsection{Instance Collection}

This section describes the procedure we adopted to gather the instances. In January 2014, we issued an online call for instances using main international mailing lists of the mathematical optimization and numerical analysis communities, reaching in this way a large set of possibly interested researchers and practitioners. The call remained open for 
ten months, during which we received a large number of contributions of different nature. The instances we gathered come both from theoretical studies as well as from real-world applications.

In addition to these spontaneous contributions, we analyzed existing generic instance libraries available containing QP instances. The libraries from which we gathered instances are

- the BARON library http://www.minlp.com/nlp-and-minlp-test-problems;

- the CUTEst library https://ccpforge.cse.rl.ac.uk/gf/project/cutest;

- the GAMS Performance libraries http://www.gamsworld.org/performance/performlib. htm;

- the MacMINLP library https://wiki.mcs.anl.gov/leyffer/index.php/MacMINLP;

- the Maros-Mészáros library http://www.doc.ic.ac.uk/ im/OOREADME.QP;

- the MINLPLib library http://www.gamsworld.org/minlp/minlplib.htm;

- the POLIP library http://polip.zib.de/pipformat.php.

Other quadratic instances were found in online libraries devoted to specific QP problems as Max-Cut, Quadratic Assignment, Portfolio Optimization, and several others. In addition, we mention that other generic libraries exist, e.g., Conic library CBLIB (http: //cblib.zib.de) and MIPLIB 2010 (http://miplib.zib.de/), to mention just a few.

At the end of this process, we had gathered more than eight thousand instances. Three quarters of them contained discrete variables, while the remainder contained only continuous variables. In more detail, we gathered $\approx 1800$ Quadratic Binary Linear $(Q B L)$ instances, $\approx 2000$ Quadratic Continuous Quadratic $(Q C Q)$ instances, and $\approx 2500$ Quadratic General Quadratic $(Q G Q)$ instances. We also received $\approx 1000$ Convex General Convex $(C G C)$ instances. We obtained relatively fewer Quadratic Binary Quadratic $(Q B Q)$, Convex Continuous Convex $(C C C)$ and Convex Mixed Convex $(C M C)$ instances, $(\approx 150, \approx 200$, and $\approx 200$ instances, respectively $)$. Finally, we found only 17 Quadratic Mixed Linear $(Q M L)$ instances. In the call for instances, no specific format requirements were imposed for the submissions.

To evaluate the instances we decided, for practical reasons, we use GAMS as common platform for all our final selection computations. For this reason, we translated all the instances we received into the GAMS format (.gms).

For each instance in this large starting set, we collected important characteristics which allowed us to classify the instances into the QP categories described in $\S 2$. As far as the variable types are concerned, we collected the following information:

- the number of binary variables;

- the number of integer variables; and

- the number of continuous variables.

If at least one binary or integer variable is present, then the instance is categorized as discrete, otherwise it is categorized as continuous. As far as the objective function is concerned, we gathered the following information: 
- the percentage of positive and negative eigenvalues of the Hessian $Q^{0}$; and

- the density of the Hessian $Q^{0}$ (number of nonzero entries divided by the total number of entries).

The number of positive (i.e., larger than $10^{-12}$ ) and negative (i.e., smaller than $-10^{-12}$ ) eigenvalues of $Q^{0}$ allowed us to identify the objective function type, as in presence of at least one negative (positive) eigenvalue the objective function is nonconvex (nonconcave). Finally, as far as the constraint types are concerned, we collected the following information:

- the number of linear constraints,

- the number of quadratic constraints,

- the number of convex constraints, and

- the number of variable bounds (for non-binary variables).

A constraint is considered quadratic if it contains at least one nonzero in a quadratic term (if present). Among the quadratic constraints, the ones whose Hessians have only non-negative eigenvalues (when $c_{u}^{i}<\infty$ ) and non-positive eigenvalues (when $c_{l}^{i}>-\infty$ ) are classified as convex constraints; thus, a quadratic constraint with two sided, finite bounds is nonconvex. Note that this might occasionally lead us to classify some instances that have conic constraints as nonconvex ones, although their feasible region is in fact convex - fortunately, only some solvers are capable of properly exploiting this property. All this information allowed us to analyse the gathered instances and to perform the filters described in the next paragraph.

\subsection{Instance Selection}

We chose instances based on the following four goals:

1. to represent as far as possible all the different categories of QP problems;

2. to gather "challenging" instances, i.e., ones which can not be easily solved by state-of-the-art solvers;

3. to include, for each of the categories, a set of well-diversified instances; and

4. to obtain a set of instances which is neither too small, so as to preserve statistical relevance, nor too large so as to being computationally manageable.

To achieve such goals, we performed the following two filters, applied in a cascade:

- First Instance Filter.

The first filter was designed to drastically reduce the number of instances by eliminating the "easy" ones. An empirical measure for the hardness of an instance is the CPU time needed by a complete solver (cf. §2.3) to solve it to global optimality. Accordingly, for each of the gathered instances we ran the complete solvers in GAMS, whose number depends on the category of the instance under consideration, cf. Table 2. Thanks to these extensive preliminary tests, we discarded all instances that are solved by at least $30 \%$ of the complete solvers within a time limit of 30 seconds. 


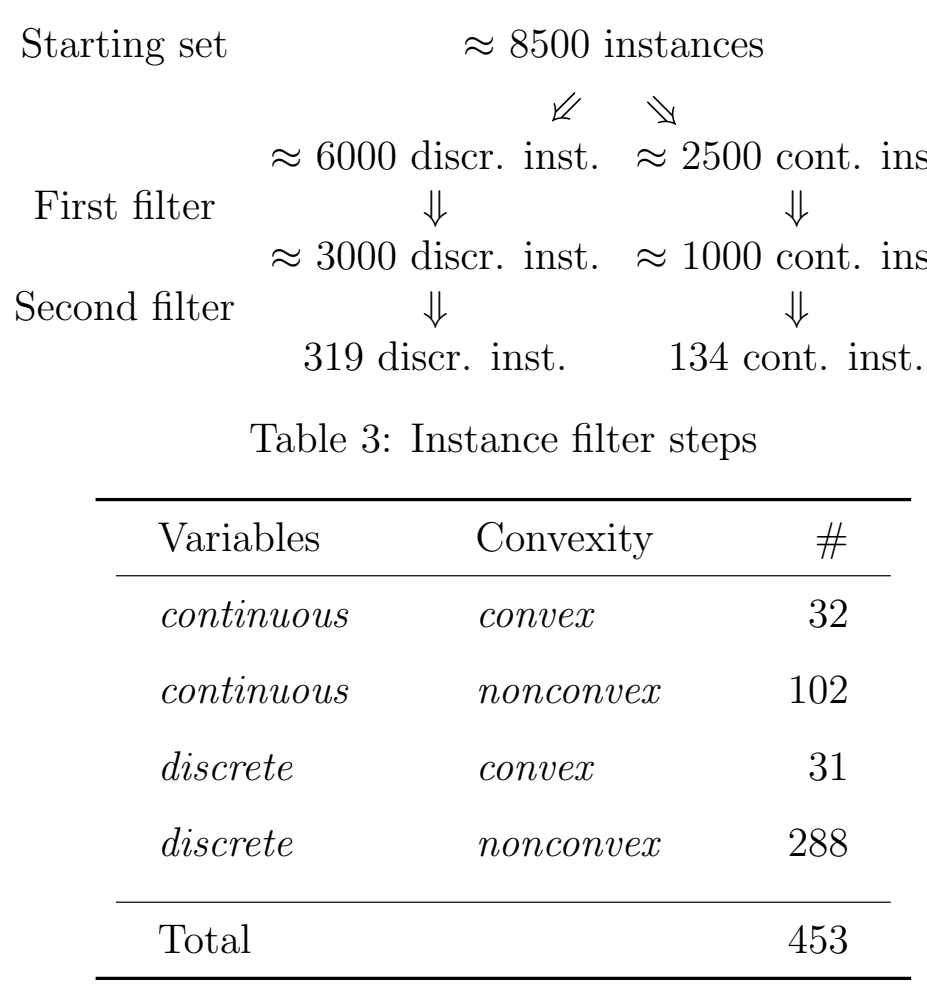

Table 4: Macro classification of the final set of instances

- Second Instance Filter.

The goal of the second filter was to eliminate "similar" instances. We carefully analyzed the instances one by one, eliminating all but a few of those with very similar size and coming from the same donor. The instances discarded by this second filter are instances of the same specific problem, e.g., we gathered many Max Cut Problem instances and we kept in the library only a representative small set of them. The selected representative instances are the larger and computationally harder ones. Finally, in order to only keep computationally challenging instances we ran a complete solver for $Q G Q$ with a time limit of 120 seconds; all the instances which have been solved to proven optimality within this time limit were discarded.

In Table 3 we summarize the two filter steps, which allowed us to identify the final set of 319 discrete instances and 134 continuous instances.

\subsection{Analysis of the Final Set of Instances}

We now analyze the features of the instances selected to be part of the library. Table 4 provides a global overview. The instances have been divided in continuous vs discrete and convex vs nonconvex, forming in this way, a classification of 4 macro categories. As previously mentioned, an instance is classified discrete if it contains at least one binary or integer variable, and continuous otherwise. On the other hand, an instance is classified as nonconvex if the objective function is nonconvex (if minimization) or nonconcave (if maximization) and/or at least one of the constraints is nonconvex, and convex otherwise.

The detailed characteristics of the instances are presented in Table 5 for discrete instances $\left({ }^{*}\{B, M, I, G\}^{*}\right)$ and in Table 6 for continuous ones $\left({ }^{*} C^{*}\right)$. For each category, the tables report the corresponding number of instances in column "\#". It can be seen 


\begin{tabular}{lllr}
\hline Obj. Fun. & Variables & Constraints & $\#$ \\
\hline \multirow{2}{*}{ Linear } & Binary & Quadratic & 9 \\
\cline { 2 - 4 } & Mixed & Convex & 14 \\
& & Quadratic & 134 \\
\cline { 2 - 4 } & Integer & Quadratic & 2 \\
\cline { 2 - 4 } Convex (if min) & General & Quadratic & 3 \\
\cline { 2 - 4 } or & Binary & Linear & 5 \\
\cline { 2 - 4 } Concave (if max) & Mixed & Linear & 12 \\
\hline \multirow{2}{*}{ Quadratic } & & Quadratic & 6 \\
\hline Total & Binary & None & 23 \\
& & Linear & 91 \\
\cline { 2 - 4 } & Mixed & Linear & 11 \\
& & Quadratic & 1 \\
\cline { 2 - 4 } & Integer & Linear & 2 \\
\cline { 2 - 4 } & General & Quadratic & 1 \\
\hline
\end{tabular}

Table 5: Classification of the final set of discrete instances

that the final set well respects the original distribution of the gathered instances among the different categories. Indeed, the discrete categories $L M Q$ and $Q B L$ are well represented by 134 and 91 instances, respectively. Similarly, the continuous categories $L C Q$ and $Q C Q$ are well represented by 52 and 30 instances, respectively. Moreover, the library actually covers the large majority of all possible categories of instances.

We now report some graphs that help in illustrating the main features of the instances. In Figure 1 (left) we plot the number of variables (horizontal axis) versus the number of constraints (vertical axis), both in logarithmic scale. Continuous instances are marked with "+" and discrete ones with " $\times$ ". Box constraints are not counted as constraints. The figure shows that the library contains a quite diverse set of instances in terms of number of variables and constraints. The record on the maximal number of variables and constraints (both $\approx 1,000,000$ ) is set by the instances QPLIB_8547 and QPLIB_9008. Figure 1 (right) plots the number of nonzero elements in the gradient of the objective function and the Jacobian and the number of these nonzeros corresponding to nonlinear variables, that is, 


\begin{tabular}{llr}
\hline Obj. Fun. & Constraints & $\#$ \\
\hline Linear & Convex & 13 \\
& Quadratic & 52 \\
\hline Convex (if min) & Box & 3 \\
or & Linear & 16 \\
Concave (if max) & Quadratic & 11 \\
\hline \multirow{2}{*}{ Quadratic } & Linear & 6 \\
& Convex & 3 \\
& Quadratic & 30 \\
\hline Total & & 134 \\
\hline
\end{tabular}

Table 6: Classification of the final set of continuous instances

it counts the appearances of variables in objectives and constraints and how often such an appearance is in a quadratic term.

Figure 2 describes how discrete and continuous variables are distributed within the instances. The instances are sorted accordingly to the total number of variables. For each instance we report the total number of variables with a "+", and the total number of discrete variables (binary or general integer) with a " $\times$ ". The pictures clearly show that instances with different percentages of integer and continuous variables are present in the library, and that these differences are well distributed across the whole spectrum of variable sizes.

Similarly, Figure 3 (left) describes how the number of linear and quadratic constraints are distributed within the instances. The instances are sorted accordingly to the total number of constraints. For each instance we report the total number of constraints with a "+" and the total number of quadratic constraints with a " $\times$ ". Also in this case, different percentages of linear and quadratic constraints are present and well-distributed across the spectrum of constraint sizes, although both medium- and large-size instances show a prevalence of lower percentages of quadratic constraints. In particular, from Figure 3 (left) we learn that while the maximum number of linear constraints exceeds 1,000,000, the maximum number of quadratic constraints tops up at 140,000. This is, however, reasonable, considering how quadratic constraints can, in general, be expected to be much more computationally challenging than linear ones, especially if nonconvex.

Figure 3 (right) shows the instances with at least one quadratic constraint sorted according to the number of quadratic constraints (vertical axis). For each instance we report the total number of constraints with a "+" and the total number of nonconvex quadratic constraints with a " $\times$ ". It can be seen that the majority of instances only have nonconvex constraints.

On the theme of nonconvexity, Figure 4 (left) focuses on the instances with a quadratic objective function, ordered by percentage of "problematic" eigenvalues in the Hessian $Q^{0}$ (vertical axis), by which we mean eigenvalues below $-10^{-12}$ in case of a minimization 

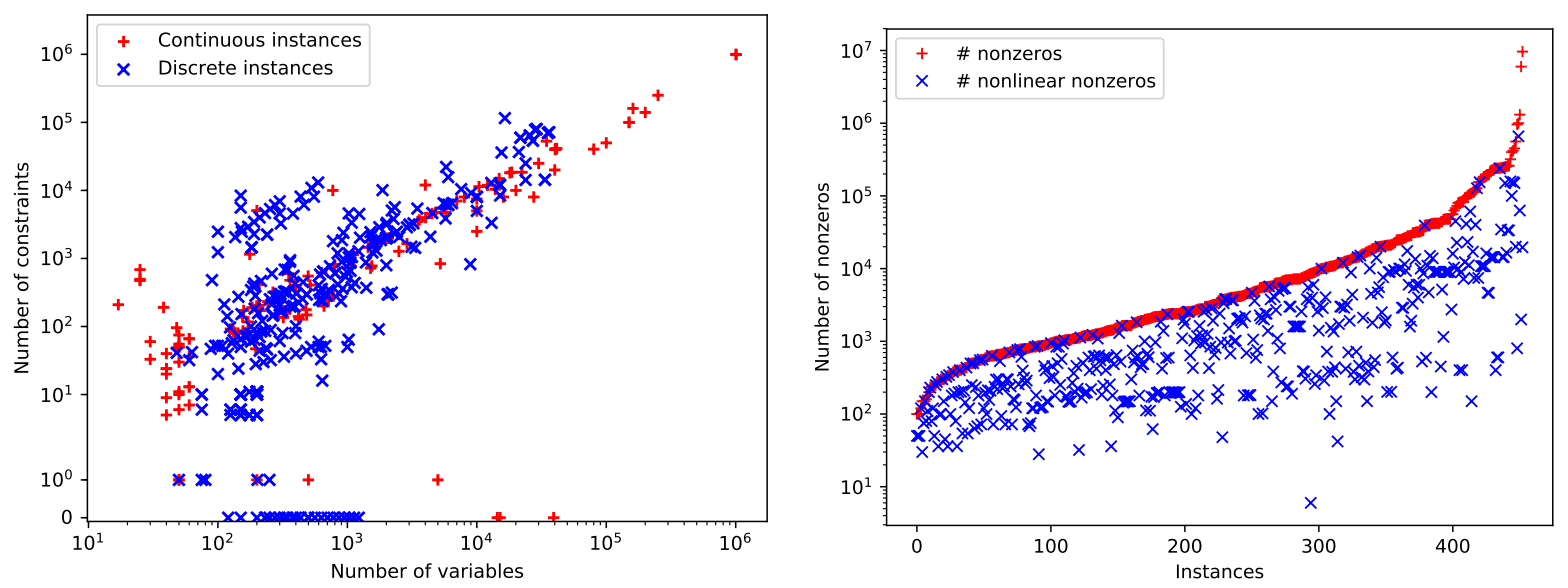

Figure 1: Distribution of number of variables and constraints of QPLIB instances (left). Number of (nonlinear) nonzeros of QPLIB instances (right).

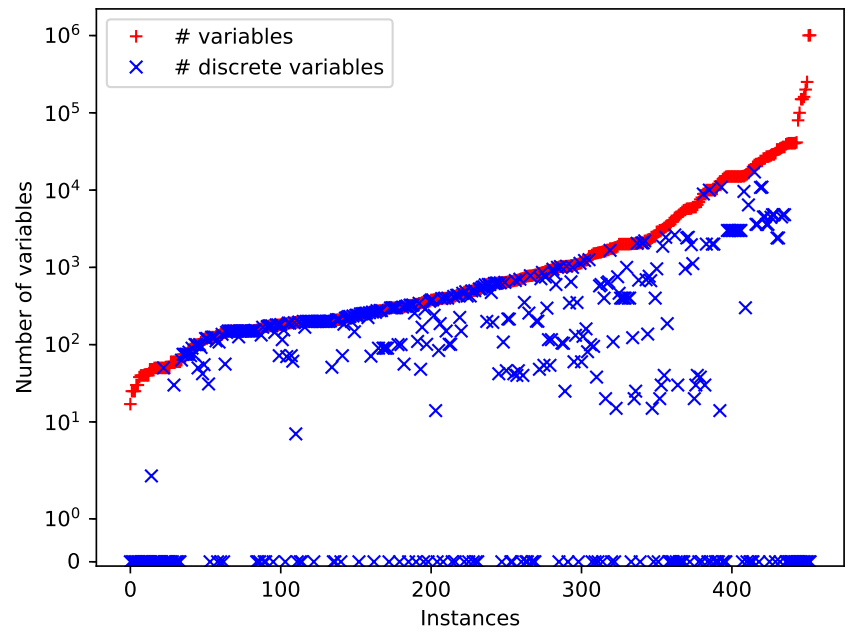

Figure 2: Number of variables of QPLIB instances.
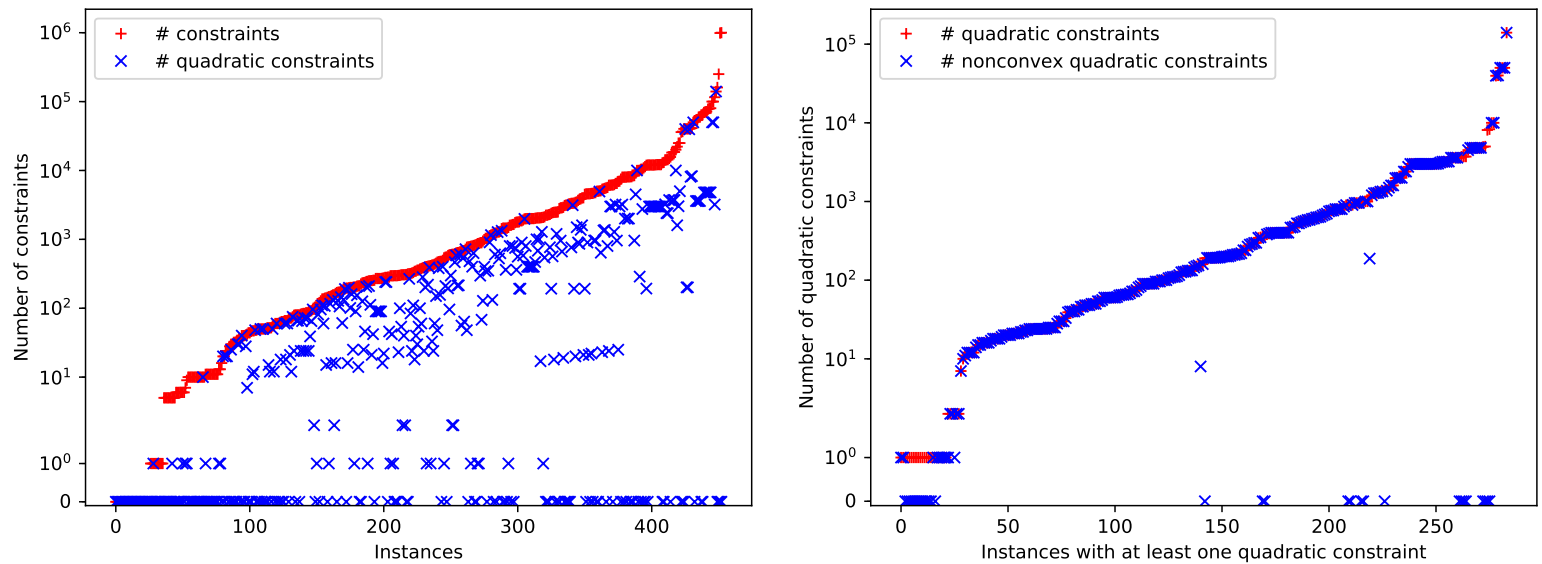

Figure 3: Number of constraints, quadratic constraints, and nonconvex quadratic constraints of QPLIB instances. 

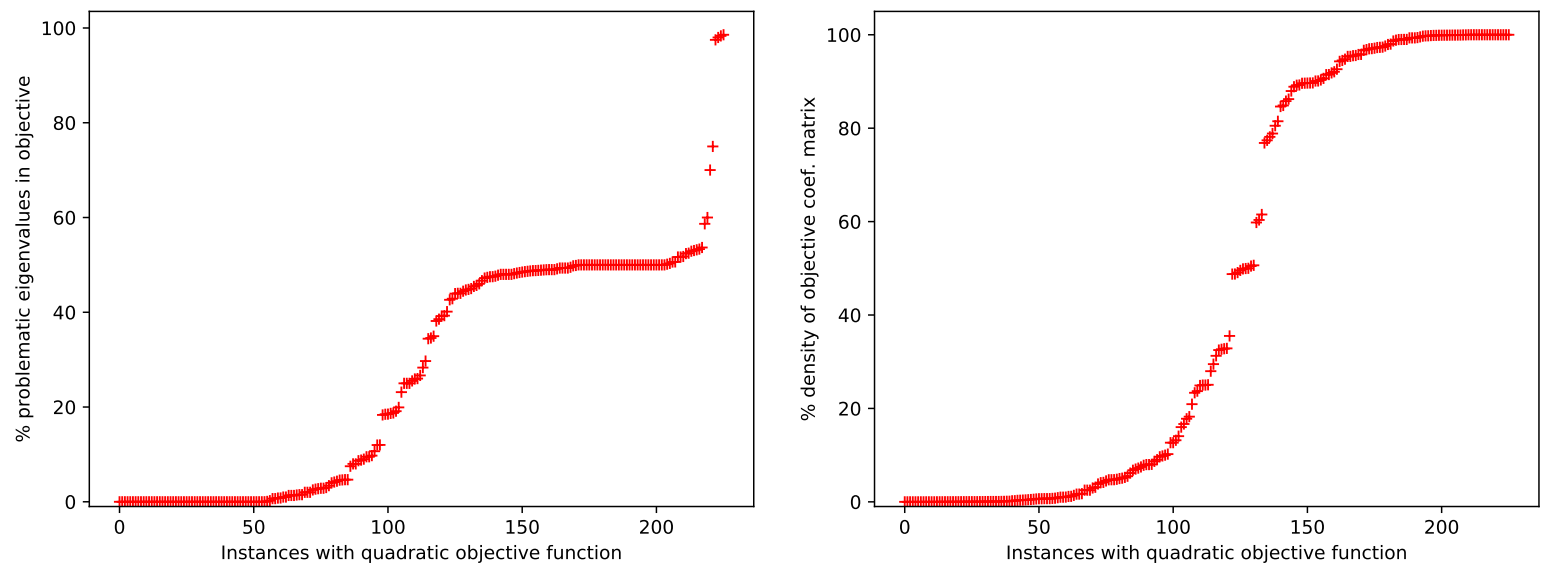

Figure 4: "Problematic" eigenvalues (left) and density (right) of the Hessian $Q^{0}$ for QPLIB instances with a quadratic objective function.

problem and eigenvalues above $10^{-12}$ in case of a maximization problem. The instances are mostly clustered around two values. About $25 \%$ of the instances have a convex (if minimization) or concave (if maximization) objective function, i.e., they have $0 \%$ of "problematic" eigenvalues. Among the others, a vast majority has around $50 \%$ of "problematic" eigenvalues. However, instances with high or low percentages of "problematic" eigenvalues are present, too.

Similarly, Figure 4 (right) shows the instances with a quadratic objective function sorted according to the density of the Hessian $Q^{0}$ (vertical axis). The majority of the instances have either a very low or a rather high density: indeed, about $30 \%$ of the instances have density smaller than $5 \%$, and about $30 \%$ of the instances have density larger than $50 \%$. However, also intermediate values are present.

Additional details on the instance features can be found in Appendix A.

\subsection{Website}

The QPLIB instances are publicly accessible at the website http://qplib.zib.de, which was created by extending scripts and tools initially developed for MINLPLib 2 [139]. We provide all instances in GAMS (.gms), AMPL (.mod), CPLEX (.lp) [77], and QPLIB (.qplib) formats. The latter is a new format specifically for QP instances. In comparison to more high level formats such as .gms and .lp, the new format offers three main advantages: it is easier to read by a stand-alone parser, it typically produces smaller files, and it permits the inclusion of two-sided inequalities without needless repetition of data. See Appendix B for more details.

Beyond the instances, the website provides a rich set of metadata for each instance: the three letter problem classification (as described in §3.3), the contributor of the instance, basic properties such as the number of variables and constraints of different types, the sense and convexity/concavity of the objective function, and information on the nonzero structure of the problem. In addition, we display a visualization of the sparsity patterns of the Jacobian and the Hessian matrix of the Lagrangian function, if the instance size allows. In the plots of the Jacobian nonzero pattern, the linear and nonlinear entries are distinguished by color. Figure 5 shows an example for instance QPLIB_2967. Finally, 

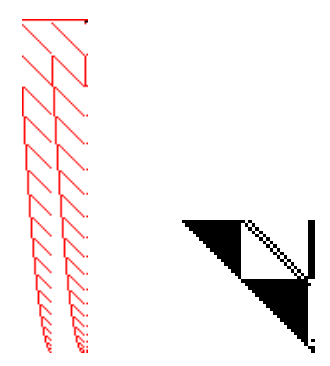

Figure 5: Example for the sparsity pattern of the Jacobian of the constraint functions (left) and of the upper-right triangle of the Hessian of the Lagrangian function (right) for instance QPLIB_2967. The gradient of the objective function is displayed as the first row of the Jacobian matrix. Non-constant entries are shown in red.

feasible solution points are provided for most instances.

The entire set of instances can be explored in a searchable and sortable table of selected instance features: problem classification, convexity of the continuous relaxation, number of (all, binary, integer) variables, (all, quadratic) constraints, nonzeros, problematic eigenvalues in $Q^{0}$, and density of $Q^{0}$. Finally, a statistics page displays diagrams on the composition of the library according to different criteria: the number of instances according to problem type, variable and constraint types, convexity, problem size, and density. A file containing the relevant metadata for each instance can be downloaded in commaseparated-values (csv) format, so that researchers can easily compile their own subset of instances according to these statistics.

The complete library can be downloaded as one archive, which contains the website for offline browsing and exploration. In the future, we plan to extend the website by references to the literature.

\section{Final Remarks}

This paper described the first comprehensive library of instances for Quadratic Programming (QP). Since QP comprises different and "varied" categories of problems, we proposed a classification and we briefly discussed the main classes of solution methods for QP. We then described the steps of the adopted process used to filter the gathered instances in order to build the new library. Our design goals were to build a library which is computationally challenging and as broad as possible, i.e., it represents the largest possible categories of QP problems, while remaining of manageable size. We also proposed a stand-alone QP format that is intended for the convenient exchange and use of our QP instances.

We want to stress once again that we intentionally avoided to perform a computational comparison of the performances of different solution methods or solver implementations. Our goal was instead to provide a broad test bed of instances for researchers and practitioners in the field. This new library will hopefully serve as a point of reference to inspire and test new ideas and algorithms for QP problems.

Finally, we want to emphasize that this QP collection can only be a snapshot of the types of problems that researchers and practitioners have worked on in the past. With the growing interest in this area, we hope that new applications and instances will become available and that the library can be extended dynamically in the future. 


\section{Acknowledgments}

We are grateful to all the donors who provided instances for the library. We gratefully acknowledge the financial support of the Gaspard Monge Program for Optimization and operations research (PGMO) and the logistic support of GAMS for having provided us with a license for their software. Finally, we would like to acknowledge the financial and networking support by the COST Action TD1207. The work of the fifth and twelfth author was supported by the Research Campus MODAL Mathematical Optimization and Data Analysis Laboratories funded by the Federal Ministry of Education and Research (BMBF Grant 05M14ZAM). The work of the sixth author was supported by the EPSRC grant EP/M025179/1. All responsibility for the content of this publication is assumed by the authors.

\section{References}

[1] T. Achterberg. SCIP: Solving Constraint Integer Programs. Mathematical Programming Computation, 1(1):1-41, 2009.

[2] Satoru Adachi, Satoru Iwata, Yuji Nakatsukasa, and Akiko Takeda. Solving the trust-region subproblem by a generalized eigenvalue problem. SIAM Journal on Optimization, 27(1):269-291, 2017.

[3] E. Ahmetović and I. E. Grossmann. Global superstructure optimization for the design of integrated process water networks. AIChE Journal, 57(2):434-457, 2011.

[4] M. Alfaki and D. Haugland. A multi-commodity flow formulation for the generalized pooling problem. Journal of Global Optimization, 56(3):917-937, 2013.

[5] E.D. Andersen, C. Roos, and T. Terlaky. On implementing a primal-dual interiorpoint method for conic quadratic optimization. Mathematical Programming, 95(2):249-277, 2003.

[6] Erling D. Andersen and Knud D. Andersen. The Mosek interior point optimizer for linear programming: An implementation of the homogeneous algorithm. In Hans Frenk, Kees Roos, Tamás Terlaky, and Shuzhong Zhang, editors, High Performance Optimization, pages 197-232. Springer US, Boston, MA, 2000.

[7] M. F. Anjos and F. Liers. Global approaches for facility layout and VLSI floorplanning. In M. F. Anjos and J. B. Lasserre, editors, Handbook on Semidefinite, Conic and Polynomial Optimization, volume 166 of International Series in Operations Research 8 Management Science, pages 849-877. Springer US, 2012.

[8] K. M. Anstreicher. Recent advances in the solution of quadratic assignment problems. Mathematical Programming, 97(1-2):27-42, 2003.

[9] C. Audet, A. Guillou, P. Hansen, F. Messine, and S. Perron. The small hexagon and heptagon with maximum sum of distances between vertices. Journal of Global Optimization, 49(3):467-480, 2011. 
[10] C. Audet, P. Hansen, and F. Messine. The small octagon with longest perimeter. Journal of Combinatorial Theory, Series A, 114(1):135-150, 2007.

[11] C. Audet, P. Hansen, and F. Messine. Simple polygons of maximum perimeter contained in a unit disk. Discrete \& Computational Geometry, 41(2):208-215, 2009.

[12] C. Audet, P. Hansen, F. Messine, and J. Xiong. The largest small octagon. Journal of Combinatorial Theory, Series A, 98(1):46-59, 2002.

[13] C. Audet and J. Ninin. Maximal perimeter, diameter and area of equilateral unitwidth convex polygons. Journal of Global Optimization, 56(3):1007-1016, 2013.

[14] M. Bagajewicz. A review of recent design procedures for water networks in refineries and process plants. Computers $\&$ Chemical Engineering, 24(9-10):2093-2113, 2000.

[15] Pietro Belotti, Jon Lee, Leo Liberti, F. Margot, and Andreas Wächter. Branching and bounds tightening techniques for non-convex MINLP. Optimization Methods and Software, 24(4-5):597-634, 2009.

[16] Michael J. Best. Quadratic programming with computer programs. Advances in applied mathematics. Chapman and Hall/CRC, Taylor \& Francis, 1st edition, 2017.

[17] A. Billionnet, S. Elloumi, and M.C. Plateau. Improving the performance of standard solvers for quadratic 0-1 programs by a tight convex reformulation: The QCR method. Discrete Applied Mathematics, 157(6):1185-1197, 2009.

[18] Alain Billionnet, Sourour Elloumi, and Amélie Lambert. An efficient compact quadratic convex reformulation for general integer quadratic programs. Computational Optimization \& Applications, 54(1):141-162, 2013.

[19] E. Robert Bixby, Mary Fenelon, Zonghao Gu, Ed Rothberg, and Roland Wunderling. MIP: Theory and practice - closing the gap. In M. J. D. Powell and S. Scholtes, editors, System Modelling and Optimization: Methods, Theory and Applications. 19th IFIP TC7 Conference on System Modelling and Optimization July 12-16, 1999, Cambridge, UK, pages 19-49. Springer US, Boston, MA, 2000.

[20] A. Bley, A. M. Gleixner, T. Koch, and S. Vigerske. Comparing MIQCP solvers to a specialised algorithm for mine production scheduling. In H. G. Bock, X. P. Hoang, R. Rannacher, and J. P. Schlöder, editors, Modeling, Simulation and Optimization of Complex Processes, pages 25-39. Springer Berlin Heidelberg, 2012.

[21] L. Blum, M. Shub, and S. Smale. On a theory of computation and complexity over the real numbers: NP-completeness, recursive functions, and universal machines. Bulletin of the American Mathematical Society, 21(1):1-46, 1989.

[22] I. M. Bomze, M. Budinich, P. M. Pardalos, and M. Pelillo. The maximum clique problem. In D.-Z. Du and P. M. Pardalos, editors, Handbook of Combinatorial Optimization, pages 1-74. Springer US, 1999. 
[23] Pierre Bonami, Lorenz T. Biegler, Andrew R. Conn, G. Cornuéjols, Ignacio E. Grossmann, Carl D. Laird, Jon Lee, Andrea Lodi, F. Margot, Nicolas Sawaya, and Andreas Wächter. An algorithmic framework for convex mixed integer nonlinear programs. Discrete Optimization, 5(2):186-204, 2008.

[24] C. Bragalli, C. D'Ambrosio, J. Lee, A. Lodi, and P. Toth. On the optimal design of water distribution networks: A practical MINLP approach. Optimization and Engineering, 13(2):219-246, 2012.

[25] Christoph Buchheim and Angelika Wiegele. Semidefinite relaxations for non-convex quadratic mixed-integer programming. Mathematical Programming, 141(1):435-452, 2013.

[26] S. Burer and A. Saxena. The MILP road to MIQCP. In Jon Lee and Sven Leyffer, editors, Mixed Integer Nonlinear Programming, volume 154 of The IMA Volumes in Mathematics and its Applications, pages 373-405. Springer, 2012.

[27] Samuel Burer. Copositive programming. In F. Miguel Anjos and B. Jean Lasserre, editors, Handbook on Semidefinite, Conic and Polynomial Optimization, pages 201218. Springer US, Boston, MA, 2012.

[28] Michael R. Bussieck and S. Vigerske. MINLP solver software. In J. J. Cochran et.al., editor, Wiley Encyclopedia of Operations Research and Management Science. Wiley \& Sons, Inc., 2010.

[29] R. H. Byrd, J. Nocedal, and R.A. Waltz. KNITRO: An integrated package for nonlinear optimization. In G. di Pillo and M. Roma, editors, Large-Scale Nonlinear Optimization, volume 83 of Nonconvex Optimization and Its Applications, pages 35-59. Springer, 2006.

[30] I. Castillo, J. Westerlund, S. Emet, and T. Westerlund. Optimization of block layout design problems with unequal areas: A comparison of MILP and MINLP optimization methods. Computers \&3 Chemical Engineering, 30(1):54 - 69, 2005.

[31] P. A. C. Castillo, V. Mahalec, and J. D. Kelly. Inventory pinch algorithm for gasoline blend planning. AIChE Journal, 59(10):3748-3766, 2013.

[32] J. Castro, A. Frangioni, and C. Gentile. Perspective Reformulations of the CTA Problem with $L_{2}$ Distances. Operations Research, 62(4):891-909, 2014.

[33] P. M. Castro and J. P. Teles. Comparison of global optimization algorithms for the design of water-using networks. Computers $\& 3$ Chemical Engineering, 52:249-261, 2013.

[34] A. R. Conn, N. I. M. Gould, D. Orban, and Ph. L. Toint. A primal-dual trustregion algorithm for non-convex nonlinear programming. Mathematical Programming, $87(2): 215-249,2000$.

[35] R. Dakin. A tree search algorithm for mixed programming problems. Computer Journal, 8(3):250-255, 1965. 
[36] C. D'Ambrosio, J. Linderoth, and J. Luedtke. Valid inequalities for the pooling problem with binary variables. In O. Günlük and G. J. Woeginger, editors, Integer Programming and Combinatoral Optimization, volume 6655 of Lecture Notes in Computer Science, pages 117-129. Springer Berlin Heidelberg, 2011.

[37] Z. Deng, Y. Bai, S.-C. Fang, Y. Tian, and W. Xing. A branch-and-cut approach to portfolio selection with marginal risk control in a linear conic programming framework. Journal of Systems Science and Systems Engineering, 22(4):385-400, 2013.

[38] Hongbo Dong. Relaxing nonconvex quadratic functions by multiple adaptive diagonal perturbations. SIAM Journal on Optimization, 26(3):1962-1985, 2016.

[39] M. C. Dorneich and N. V. Sahinidis. Global optimization algorithms for chip layout and compaction. Engineering Optimization, 25(2):131-154, 1995.

[40] Z. Dostál. Optimal Quadratic Programming Algorithms: With Applications to Variational Inequalities. Springer Verlag, Heidelberg, Berlin, New York, 2009.

[41] Arne Drud. CONOPT: A GRG code for large sparse dynamic nonlinear optimization problems. Mathematical Programming, 31(2):153-191, 1985.

[42] Arne Stolbjerg Drud. CONOPT - a large-scale GRG code. INFORMS Journal on Computing, 6(2):207-216, 1994.

[43] Arne Stolbjerg Drud. SBB. ARKI Consulting and Development A/S, 2017.

[44] Mirjam Dür. Copositive programming - a survey. In Moritz Diehl, Francois Glineur, Elias Jarlebring, and Wim Michiels, editors, Recent Advances in Optimization and its Applications in Engineering: The 14th Belgian-French-German Conference on Optimization, pages 3-20. Springer, Berlin, Heidelberg, 2010.

[45] Marco A. Duran and Ignacio E. Grossmann. An outer-approximation algorithm for a class of mixed-integer nonlinear programs. Mathematical Programming, 36(3):307339, 1986.

[46] Jennifer B. Erway and Philip E. Gill. A subspace minimization method for the trust-region step. SIAM Journal on Optimization, 20(3):1439-1461, 2010.

[47] D. C. Faria and M. J. Bagajewicz. A new approach for global optimization of a class of MINLP problems with applications to water management and pooling problems. AIChE Jornal, 58(8):2320-2335, 2012.

[48] FICO. Xpress optimization suite, 2017.

[49] R. Fletcher. Stable reduced Hessian updates for indefinite quadratic programming. Mathematical Programming, 87(2):251-264, 2000.

[50] C.A. Floudas and V. Visweswaran. A global optimization algorithm (GOP) for certain classes of nonconvex NLPs - I. Theory. Computers \& Chemical Engineering, 14(12):1397-1417, 1990. 
[51] A. Frangioni, F. Furini, and C. Gentile. Approximated Perspective Relaxations: a Project \& Lift Approach. Computational Optimization and Applications, 63(3):705$735,2016$.

[52] A. Frangioni, L. Galli, and M.G. Scutellà. Delay-Constrained Shortest Paths: Approximation Algorithms and Second-Order Cone Models. Journal of Optimization Theory and Applications, 164(3):1051-1077, 2015.

[53] A. Frangioni, L. Galli, and G. Stea. Delay-constrained routing problems: Accurate scheduling models and admission control. Computers $\mathcal{G}$ Operations Research, 81:67$77,2017$.

[54] A. Frangioni and C. Gentile. Perspective Cuts for a Class of Convex 0-1 Mixed Integer Programs. Mathematical Programming, 106(2):225-236, 2006.

[55] A. Frangioni and C. Gentile. SDP Diagonalizations and Perspective Cuts for a Class of Nonseparable MIQP. Operations Research Letters, 35(2):181-185, 2007.

[56] A. Frangioni and C. Gentile. A Computational Comparison of Reformulations of the Perspective Relaxation: SOCP vs. Cutting Planes. Operations Research Letters, 37(3):206-210, 2009.

[57] A. Frangioni, C. Gentile, E. Grande, and A. Pacifici. Projected Perspective Reformulations with Applications in Design Problems. Operations Research, 59(5):1225-1232, 2011.

[58] B. Geissler, A. Morsi, and L. Schewe. A new algorithm for MINLP applied to gas transport energy cost minimization. In M. Jünger and G. Reinelt, editors, Facets of Combinatorial Optimization, pages 321-353. Springer Berlin Heidelberg, 2013.

[59] I. Gentilini, F. Margot, and K. Shimada. The travelling salesman problem with neighbourhoods: MINLP solution. Optimization Methods and Software, 28(2):364$378,2013$.

[60] E. M. Gertz and S. J. Wright. Object-oriented software for quadratic programming. ACM Transactions on Mathematical Software, 29(1):58-81, 2003.

[61] P. E. Gill, W. Murray, and M. A. Saunders. SNOPT: An SQP algorithm for largescale constrained optimization. SIAM Journal on Optimization, 12(4):979-1006, 2002 .

[62] P. E. Gill, W. Murray, and M. A. Saunders. SNOPT: An SQP algorithm for large-scale constrained optimization. SIAM Review, 47(1):99-131, 2005.

[63] Philip E. Gill and Elizabeth Wong. Methods for convex and general quadratic programming. Mathematical Programming Computation, 7(1):71-112, 2015.

[64] A. M. Gleixner, H. Held, W. Huang, and S. Vigerske. Towards globally optimal operation of water supply networks. Numerical Algebra, Control and Optimization, 2(4):695-711, 2012. 
[65] N. I. M. Gould, D. Orban, and D. P. Robinson. Trajectory-following methods for large-scale degenerate convex quadratic programming. Mathematical Programming Computation, 5(2):113-142, 2013.

[66] N. I. M. Gould, D. Orban, and Ph. L. Toint. GALAHAD, a library of thread-safe Fortran 90 packages for large-scale nonlinear optimization. ACM Transactions on Mathematical Software, 29(4):353-372, 2003.

[67] N. I. M. Gould and D. P. Robinson. A dual gradient-projection method for large-scale strictly convex quadratic problems. Computational Optimization and Applications, 67(1):1-38, 2017.

[68] N. I. M. Gould and Ph. L. Toint. A quadratic programming bibliography. Numerical Analysis Group Internal Report 2000-1, Rutherford Appleton Laboratory, Chilton, Oxfordshire, England, 2000.

[69] N. I. M. Gould and Ph. L. Toint. An iterative working-set method for large-scale nonconvex quadratic programming. Applied Numerical Mathematics, 43(1-2):109-128, 2002.

[70] Nicholas I. M. Gould, Stefano Lucidi, Massimo Roma, and Philippe L. Toint. Solving the trust-region subproblem using the Lanczos method. SIAM Journal on Optimization, 9(2):504-525, 1999.

[71] Nicholas I. M. Gould, Daniel P. Robinson, and H. Sue Thorne. On solving trust-region and other regularised subproblems in optimization. Mathematical Programming Computation, 2(1):21-57, Mar 2010.

[72] C. E. Gounaris, E. L. First, and C. A. Floudas. Estimation of diffusion anisotropy in microporous crystalline materials and optimization of crystal orientation in membranes. The Journal of Chemical Physics, 139(12):124703, 2013.

[73] William W. Hager. Minimizing a quadratic over a sphere. SIAM Journal on Optimization, 12(1):188-208, 2001.

[74] M. M. F. Hasan, I. A. Karimi, and C. M. Avison. Preliminary synthesis of fuel gas networks to conserve energy and preserve the environment. Industrial $\&$ Engineering Chemistry Research, 50(12):7414-7427, 2011.

[75] Raymond Hemmecke, Matthias Köppe, Jon Lee, and Robert Weismantel. Nonlinear integer programming. In Michael Jünger, M. Thomas Liebling, Denis Naddef, L. George Nemhauser, R. William Pulleyblank, Gerhard Reinelt, Giovanni Rinaldi, and A. Laurence Wolsey, editors, 50 Years of Integer Programming 1958-2008: From the Early Years to the State-of-the-Art, pages 561-618. Springer Berlin Heidelberg, 2010.

[76] M. Hifi and R. M'Hallah. A literature review on circle and sphere packing problems: Models and methodologies. Advances in Operations Research, 2009, 2009.

[77] IBM ILOG. CPLEX Optimization Studio, 12.7.0 edition, 2016. 
[78] R. Jeroslow. There cannot be any algorithm for integer programming with quadratic constraints. Operations Research, 21(1):221-224, 1973.

[79] J. Jeżowski. Review of water network design methods with literature annotations. Industrial $\&$ Engineering Chemistry Research, 49(10):4475-4516, 2010.

[80] J Kallrath. Exact computation of global minima of a nonconvex portfolio optimization problem. In C. A. Floudas and P. M. Pardalos, editors, Frontiers in Global Optimization, pages 237-254. Kluwer Academic Publishers, 2003.

[81] J. Kallrath. Cutting circles and polygons from area-minimizing rectangles. Journal of Global Optimization, 43(2-3):299-328, 2009.

[82] J. Kallrath and S. Rebennack. Cutting ellipses from area-minimizing rectangles. Journal of Global Optimization, 59(2-3):405-437, 2014.

[83] C. S. Khor, B. Chachuat, and N. Shah. Fixed-flowrate total water network synthesis under uncertainty with risk management. Journal of Cleaner Production, 77:79-93, 2014.

[84] Thorsten Koch, Tobias Achterberg, Erling Andersen, Oliver Bastert, Timo Berthold, Robert E. Bixby, Emilie Danna, Gerald Gamrath, Ambros M. Gleixner, Stefan Heinz, Andrea Lodi, Hans Mittelmann, Ted Ralphs, Domenico Salvagnin, Daniel E. Steffy, and Kati Wolter. MIPLIB 2010. Mathematical Programming Computation, 3(2):103-163, 2011.

[85] Gary Kochenberger, Jin-Kao Hao, Fred Glover, Mark Lewis, Zhipeng Lü, Haibo Wang, and Yang Wang. The unconstrained binary quadratic programming problem: a survey. Journal of Combinatorial Optimization, 28(1):58-81, 2014.

[86] Gary R. Kocis and Ignacio E. Grossmann. Computational experience with DICOPT solving MINLP problems in process systems engineering. Computers $\&$ Chemical Engineering, 13(3):307-315, 1989.

[87] S. P. Kolodziej, P. M. Castro, and I. E. Grossmann. Global optimization of bilinear programs with a multiparametric disaggregation technique. Journal of Global Optimization, 57(4):1039-1063, 2013.

[88] S. P. Kolodziej, I. E. Grossmann, K. C. Furman, and N. W. Sawaya. A discretizationbased approach for the optimization of the multiperiod blend scheduling problem. Computers \& Chemical Engineering, 53:122-142, 2013.

[89] Nathan Krislock, Jérôme Malick, and Frédéric Roupin. BiqCrunch: A semidefinite branch-and-bound method for solving binary quadratic problem. ACM Transactions on Mathematical Software, 43(4):32:1-32:23, January 2017.

[90] A. Land and A. Doig. An automatic method of solving discrete programming problems. Econometrica, 28(3):497-520, 1960.

[91] Leon Lasdon, John Plummer, Zsolt Ugray, and Michael Bussieck. Improved filters and randomized drivers for multi-start global optimization. McCombs Research Paper Series IROM-06-06, McCombs School of Business, 2006. 
[92] G.M. Lee, N.N. Tam, and N.D. Yen. Quadratic Programming and Affine Variational Inequalities: A Qualitative Study. Nonconvex Optimization and Its Applications. Springer US, 2006.

[93] J. Li, A. Li, I. A. Karimi, and R. Srinivasan. Improving the robustness and efficiency of crude scheduling algorithms. AIChE Journal, 53(10):2659-2680, 2007.

[94] J. Li, R. Misener, and C. A. Floudas. Continuous-time modeling and global optimization approach for scheduling of crude oil operations. AIChE Journal, 58(1):205-226, 2012.

[95] J. Li, R. Misener, and C. A. Floudas. Scheduling of crude oil operations under demand uncertainty: A robust optimization framework coupled with global optimization. AIChE Journal, 58(8):2373-2396, 2012.

[96] X. Li, E. Armagan, A. Tomasgard, and P. I. Barton. Stochastic pooling problem for natural gas production network design and operation under uncertainty. AIChE Journal, 57(8):2120-2135, 2011.

[97] X. Li, A. Tomasgard, and P. I. Barton. Decomposition strategy for the stochastic pooling problem. Journal of Global Optimization, 54(4):765-790, 2012.

[98] X. Lin, C. A. Floudas, and J. Kallrath. Global solution approach for a nonconvex MINLP problem in product portfolio optimization. Journal of Global Optimization, 32(3):417-431, 2005.

[99] Y. Lin and L. Schrage. The global solver in the LINDO API. Optimization Methods and Software, 24(4-5):657-668, 2009.

[100] E. M. Loiola, N. M. Maia de Abreu, P. O. Boaventura-Netto, P. Hahn, and T. Querido. A survey for the quadratic assignment problem. European Journal of Operational Research, 176(2):657-690, 2007.

[101] C. D. Maranas, I. P. Androulakis, C. A. Floudas, A. J. Berger, and J. M. Mulvey. Solving long-term financial planning problems via global optimization. Journal of Economic Dynamics and Control, 21(8-9):1405-1425, 1997.

[102] R. Misener and C. A. Floudas. Advances for the pooling problem: Modeling, global optimization, and computational studies. Applied and Computational Mathematics, $8(1): 3-22,2009$.

[103] R. Misener and C. A. Floudas. Global optimization of large-scale pooling problems: Quadratically constrained MINLP models. Industrial \& Engineering Chemistry Research, 49(11):5424-5438, 2010.

[104] Ruth Misener and Christodoulos A. Floudas. GloMIQO: Global Mixed-Integer Quadratic Optimizer. Journal of Global Optimization, 57(1):3-50, 2013.

[105] Ruth Misener and Christodoulos A. Floudas. ANTIGONE: Algorithms for coNTinuous / Integer Global Optimization of Nonlinear Equations. Journal of Global Optimization, 59(2-3):503-526, 2014. 
[106] S. Mouret, I. E. Grossmann, and P. Pestiaux. A novel priority-slot based continuoustime formulation for crude-oil scheduling problem. Industrial \& Engineering Chemistry Research, 48(18):8515-8528, 2009.

[107] S. Mouret, I. E. Grossmann, and P. Pestiaux. A new Lagrangian decomposition approach applied to the integration of refinery planning and crude-oil scheduling. Computers \& Chemical Engineering, 35(12):2750-2766, 2011.

[108] B. A. Murtagh and M. A. Saunders. Large-scale linearly constrained optimization. Mathematical Programming, 14(1):41-72, 1978.

[109] B. A. Murtagh and M. A. Saunders. A projected Lagrangian algorithm and its implementation for sparse nonlinear constraints. In A. G. Buckley and J.-L. Goffin, editors, Algorithms for Constrained Minimization of Smooth Nonlinear Functions, volume 16 of Mathematic Programming Studies, pages 84-117. Springer, Berlin, Heidelberg, 1982.

[110] A. Neumaier. Complete search in continuous global optimization and constraint satisfaction. Acta Numerica, 13:271-369, 2004.

[111] A. Nyberg, I. E. Grossmann, and T. Westerlund. The optimal design of a threeechelon supply chain with inventories under uncertainty, 2012.

[112] D. J. Papageorgiou, A. Toriello, G. L. Nemhauser, and M. W. P. Savelsbergh. Fixedcharge transportation with product blending. Transportation Science, 46(2):281-295, 2012.

[113] P. Parpas and B. Rustem. Global optimization of the scenario generation and portfolio selection problems. In M. Gavrilova, O. Gervasi, V. Kumar, C. Tan, D. Taniar, A. Laganá, Y. Mun, and H. Choo, editors, Computational Science and Its Applications - ICCSA 2006, volume 3982 of Lecture Notes in Computer Science, pages 908-917. Springer-Verlag, 2006.

[114] V. Pham, C. Laird, and M. El-Halwagi. Convex hull discretization approach to the global optimization of pooling problems. Industrial \& Engineering Chemistry Research, 48(4):1973-1979, 2009.

[115] G. Di Pillo, L. Grippo, and F. Lampariello. A class of structured quasi-newton algorithms for optimal control problems. IFAC Proceedings Volumes, 16(8):101107, 1983. 4th IFAC Workshop on Applications of Nonlinear Programming to Optimization and Control, San Francisco, CA, USA, 20-21 June 1983.

[116] János D. Pintér. LGO - a program system for continuous and Lipschitz global optimization. In Immanuel M. Bomze, Tibor Csendes, Reiner Horst, and Panos M. Pardalos, editors, Developments in Global Optimization, pages 183-197. Springer US, Boston, MA, 1997.

[117] János D. Pintér. A model development system for global optimization. In Renato De Leone, Almerico Murli, Panos M. Pardalos, and Gerardo Toraldo, editors, High Performance Algorithms and Software in Nonlinear Optimization, pages 301-314. Springer US, Boston, MA, 1998. 
[118] J. M. Ponce-Ortega, M. M. El-Halwagi, and A. Jiménez-Gutiérrez. Global optimization for the synthesis of property-based recycle and reuse networks including environmental constraints. Computers \& Chemical Engineering, 34(3):318-330, 2010.

[119] S. Rebennack, J. Kallrath, and P. M. Pardalos. Column enumeration based decomposition techniques for a class of non-convex MINLP problems. Journal of Global Optimization, 43(2-3):277-297, 2009.

[120] Franz Rendl, Giovanni Rinaldi, and Angelika Wiegele. Solving max-cut to optimality by intersecting semidefinite and polyhedral relaxations. Mathematical Programming, 121(2):307-335, 2008.

[121] Franz Rendl and Henry Wolkowicz. A semidefinite framework for trust region subproblems with applications to large scale minimization. Mathematical Programming, 77(1):273-299, Apr 1997.

[122] L. M. Rios and N. V. Sahinidis. Portfolio optimization for wealth-dependent risk preferences. Annals of Operations Research, 177(1):63-90, 2010.

[123] E. Rothberg. Solving quadratically-constrained models using Gurobi, 2012.

[124] J. P. Ruiz and I. E. Grossmann. Exploiting vector space properties to strengthen the relaxation of bilinear programs arising in the global optimization of process network. Optimization Letters, 5(1):1-11, 2011.

[125] M. Ruiz, O. Briant, J.-M. Clochard, and B. Penz. Large-scale standard pooling problems with constrained pools and fixed demands. Journal of Global Optimization, 56(3):939-956, 2013.

[126] Y. Saif, A. Elkamel, and M. Pritzker. Global optimization of reverse osmosis network for wastewater treatment and minimization. Industrial $\& 3$ Engineering Chemistry Research, 47(9):3060-3070, 2008.

[127] K. Schittkowski. Numerical solution of a time-optimal parabolic boundary-value control problem. Journal of Optimization Theory and Applications, 27(2):271-290, Feb 1979 .

[128] Srdjan Stojanovic. Optimal damping control and nonlinear elliptic systems. SIAM Journal on Control and Optimization, 29(3):594-608, 1991.

[129] P. G. Szabó, C. M. Markót, and T. Csendes. Global optimization in geometry - circle packing into the square. In C. Audet, P. Hansen, and G. Savard, editors, Essays and Surveys in Global Optimization, pages 233-265. Springer, New York, 2005.

[130] B. Tadayon and J. C. Smith. Algorithms for an integer multicommodity network flow problem with node reliability considerations. Journal of Optimization Theory and Applications, 161(2):506-532, 2013.

[131] M. Tahanan, W. van Ackooij, A. Frangioni, and F. Lacalandra. Large-scale Unit Commitment under uncertainty. 4OR, 13(2):115-171, 2015. 
[132] A. Tarski. A decision method for elementary algebra and geometry. Technical Report R-109, Rand Corporation, 1951.

[133] Mohit Tawarmalani and Nikolaos V. Sahinidis. Convexification and Global Optimization in Continuous and Mixed-Integer Nonlinear Programming: Theory, Algorithms, Software, and Applications, volume 65 of Nonconvex Optimization and Its Applications. Kluwer Academic Publishers, 2002.

[134] Mohit Tawarmalani and Nikolaos V. Sahinidis. Global optimization of mixedinteger nonlinear programs: A theoretical and computational study. Mathematical Programming, 99(3):563-591, 2004.

[135] Mohit Tawarmalani and Nikolaos V. Sahinidis. A polyhedral branch-and-cut approach to global optimization. Mathematical Programming, 103(2):225-249, 2005.

[136] J. P. Teles, P. M. Castro, and H. A. Matos. Global optimization of water networks design using multiparametric disaggregation. Computers 85 Chemical Engineering, 40:132-147, 2012.

[137] Z. Ugray, L. Lasdon, J. Plummer, F. Glover, J. Kelly, and R. Martí. Scatter search and local NLP solvers: A multistart framework for global optimization. INFORMS Journal on Computing, 19(3):328-340, 2007.

[138] S. Vavasis. Quadratic programming is in NP. Information Processing Letters, 36:73-77, 1990.

[139] Stefan Vigerske. MINLPLib 2. In L. G. Casado, I. García, and E. M. T. Hendrix, editors, Proceedings of the XII global optimization workshop MAGO 2014, pages 137-140, 2014.

[140] Stefan Vigerske and Ambros Gleixner. SCIP: Global optimization of mixed-integer nonlinear programs in a branch-and-cut framework. Optimization Methods and Software, 33(3):563-593, 2018.

[141] J. Viswanathan and Ignacio E. Grossmann. A combined penalty function and outer-approximation method for MINLP optimization. Computers \& Chemical Engineering, 14(7):769-782, 1990.

[142] Andreas Wächter and Lorenz T. Biegler. On the implementation of a primal-dual interior point filter line search algorithm for large-scale nonlinear programming. Mathematical Programming, 106(1):25-57, 2006.

[143] Tapio Westerlund and K. Lundquist. Alpha-ECP, version 5.04. an interactive MINLPsolver based on the extended cutting plane method. Technical Report 01-178-A, Process Design Laboratory, Åbo Akademi University, Åbo, Finland, 2003.

[144] Tapio Westerlund and Ray Pörn. Solving pseudo-convex mixed integer optimization problems by cutting plane techniques. Optimization and Engineering, 3(3):253-280, 2002.

[145] Wikipedia. Quadratic programming, 2016. 
[146] Wikipedia. Quadratically constrained quadratic program, 2016.

[147] S.J. Wright. Primal-Dual Interior-Point Method. SIAM, Philadelphia, 1997.

\section{A. Instance Details}

Table 7 provides detailed data on all the instances of the final library. Column "name" is the name of the instance with the prefix "QPLIB_" stripped. Column "type" is the classification of the instance according to the taxonomy from $\S 2.2 .1$. Column "\% p.e." provides the fraction of problematic eigenvalues of $Q^{0}$, the coefficient matrix of the objective function: a positive number implies that the instance is a $Q^{* *}$, " 0.0 " implies that the instance is a $C^{* *}$, a blank implies that $Q^{0}=0$, i.e., the objective function is linear (hence, the instance is a $L^{* *}$ ). Column "\% d." describes the density of the $Q^{0}$ matrix: a blank implies that the corresponding instance has a linear objective function. For both columns ("\% p.e." and "\% d."), nonzeros values below 0.1 were rounded up to 0.1 . The following three columns describe the variables by reporting the number of binary ones ("\# b."), general integer ones ("\# i."), and continuous ones ("\# c."). Finally, the last four columns describe the constraints reporting the number of linear ones ("\# l."), nonconvex quadratic ones ("\# q."), convex quadratic ones ("\# c."), and variable bounds ("\# v."). The numbering of the instances reflects the initial order in which they were gathered. Due to our filtering this numbering is not consecutive.

Table 7: Features of QPLIB instances.

\begin{tabular}{|c|c|c|c|c|c|c|c|c|c|c|}
\hline \multirow[b]{2}{*}{ name } & \multirow[b]{2}{*}{ type } & \multicolumn{2}{|c|}{$Q^{0}$} & \multicolumn{3}{|c|}{ Variables } & \multicolumn{4}{|c|}{ Constraints } \\
\hline & & \% p.e. & $\%$ d. & \# b. & \# i. & \# c. & \# 1 & \# q. & \# c. & \# v. \\
\hline 0018 & $\mathrm{QCL}$ & 48.0 & 100.0 & 0 & 0 & 50 & 1 & 0 & 0 & 50 \\
\hline 0031 & QML & 18.3 & 99.8 & 30 & 0 & 30 & 32 & 0 & 0 & 30 \\
\hline 0032 & QML & 25.0 & 99.9 & 50 & 0 & 50 & 52 & 0 & 0 & 50 \\
\hline 0067 & QBL & 47.5 & 88.9 & 80 & 0 & 0 & 1 & 0 & 0 & 0 \\
\hline 0343 & QCL & 48.0 & 100.0 & 0 & 0 & 50 & 1 & 0 & 0 & 100 \\
\hline 0633 & QBL & 58.7 & 98.7 & 75 & 0 & 0 & 1 & 0 & 0 & 0 \\
\hline 0678 & LMQ & & & 9600 & 0 & 5537 & 7457 & 960 & 0 & 1474 \\
\hline 0681 & LMQ & & & 72 & 0 & 143 & 419 & 48 & 0 & 200 \\
\hline 0682 & LMQ & & & 71 & 0 & 190 & 501 & 96 & 0 & 296 \\
\hline 0684 & LMQ & & & 101 & 0 & 260 & 815 & 128 & 0 & 408 \\
\hline 0685 & LMQ & & & 256 & 0 & 519 & 1603 & 192 & 0 & 728 \\
\hline 0686 & LMQ & & & 692 & 0 & 1512 & 4440 & 640 & 0 & 2200 \\
\hline 0687 & LMQ & & & 672 & 0 & 1651 & 4875 & 800 & 0 & 2520 \\
\hline 0688 & LMQ & & & 1964 & 0 & 3824 & 20568 & 1600 & 0 & 6256 \\
\hline 0689 & LMQ & & & 756 & 0 & 1112 & 9800 & 288 & 0 & 1608 \\
\hline 0690 & LMQ & & & 6428 & 0 & 10048 & 112400 & 3200 & 0 & 17376 \\
\hline 0696 & LMQ & & & 187 & 0 & 207 & 390 & 33 & 0 & 260 \\
\hline 0698 & LMQ & & & 55 & 0 & 63 & 126 & 15 & 0 & 56 \\
\hline 0752 & QBL & 50.0 & 10.0 & 250 & 0 & 0 & 1 & 0 & 0 & 0 \\
\hline 0911 & QCQ & 44.0 & 50.5 & 0 & 0 & 50 & 0 & 50 & 0 & 100 \\
\hline 0975 & $\mathrm{QCQ}$ & 50.0 & 50.6 & 0 & 0 & 50 & 0 & 10 & 0 & 100 \\
\hline 1055 & QCQ & 50.0 & 100.0 & 0 & 0 & 40 & 0 & 20 & 0 & 80 \\
\hline 1143 & $\mathrm{QCQ}$ & 50.0 & 97.1 & 0 & 0 & 40 & 4 & 20 & 0 & 80 \\
\hline 1157 & QCQ & 25.0 & 94.5 & 0 & 0 & 40 & 8 & 1 & 0 & 80 \\
\hline 1353 & QCQ & 26.0 & 95.8 & 0 & 0 & 50 & 5 & 1 & 0 & 100 \\
\hline 1423 & $\mathrm{QCQ}$ & 75.0 & 95.4 & 0 & 0 & 40 & 4 & 20 & 0 & 80 \\
\hline 1437 & QCQ & 50.0 & 95.6 & 0 & 0 & 50 & 10 & 1 & 0 & 100 \\
\hline 1451 & QCQ & 50.0 & 49.1 & 0 & 0 & 60 & 6 & 60 & 0 & 120 \\
\hline 1493 & $\mathrm{QCQ}$ & 50.0 & 97.3 & 0 & 0 & 40 & 4 & 1 & 0 & 80 \\
\hline 1507 & QCQ & 26.7 & 95.8 & 0 & 0 & 30 & 3 & 30 & 0 & 60 \\
\hline 1535 & $\mathrm{QCQ}$ & 50.0 & 94.3 & 0 & 0 & 60 & 6 & 60 & 0 & 120 \\
\hline 1619 & QCQ & 50.0 & 95.5 & 0 & 0 & 50 & 5 & 25 & 0 & 100 \\
\hline 1661 & QCQ & 50.0 & 95.4 & 0 & 0 & 60 & 12 & 1 & 0 & 120 \\
\hline 1675 & QCQ & 51.7 & 48.8 & 0 & 0 & 60 & 12 & 1 & 0 & 120 \\
\hline 1703 & QCQ & 51.7 & 97.9 & 0 & 0 & 60 & 6 & 30 & 0 & 120 \\
\hline 1745 & QCQ & 50.0 & 48.8 & 0 & 0 & 50 & 5 & 50 & 0 & 100 \\
\hline 1773 & $\mathrm{QCQ}$ & 50.0 & 94.8 & 0 & 0 & 60 & 6 & 1 & 0 & 120 \\
\hline 1886 & QCQ & 50.0 & 50.0 & 0 & 0 & 50 & 0 & 50 & 0 & 100 \\
\hline 1913 & QCQ & 50.0 & 24.9 & 0 & 0 & 48 & 0 & 48 & 0 & 96 \\
\hline 1922 & $\mathrm{QCQ}$ & 50.0 & 49.6 & 0 & 0 & 30 & 0 & 60 & 0 & 60 \\
\hline 1931 & QCQ & 50.0 & 49.9 & 0 & 0 & 40 & 0 & 40 & 0 & 80 \\
\hline 1940 & QCQ & 50.0 & 25.0 & 0 & 0 & 48 & 0 & 96 & 0 & 96 \\
\hline 1967 & $\mathrm{QCQ}$ & 50.0 & 99.8 & 0 & 0 & 50 & 0 & 75 & 0 & 100 \\
\hline 1976 & QBQ & 38.2 & 7.0 & 152 & 0 & 0 & 136 & 16 & 0 & 0 \\
\hline
\end{tabular}


Table 7: Features of QPLIB instances (continued).

\begin{tabular}{|c|c|c|c|c|c|}
\hline & & & & & ariabl \\
\hline name & type & $\%$ p.e. & $\%$ d. & \# b. & \# i. \\
\hline 2017 & QBQ & 39.3 & 5.5 & 252 & 0 \\
\hline 2022 & QBQ & 38.5 & 5.2 & 275 & 0 \\
\hline 2029 & QBQ & 40.1 & 5.1 & 299 & 0 \\
\hline 2036 & QBQ & 39.2 & 4.8 & 324 & 0 \\
\hline 2047 & LBQ & & & 136 & 0 \\
\hline 2055 & LBQ & & & 153 & 0 \\
\hline 2060 & LBQ & & & 171 & 0 \\
\hline 2067 & LBQ & & & 190 & 0 \\
\hline 2073 & LBQ & & & 210 & 0 \\
\hline 2077 & LBQ & & & 231 & 0 \\
\hline 2085 & LBQ & & & 253 & 0 \\
\hline 2087 & LBQ & & & 276 & 0 \\
\hline 2096 & LBQ & & & 300 & 0 \\
\hline 2165 & LMQ & & & 683 & 0 \\
\hline 2166 & LMQ & & & 345 & 0 \\
\hline 2167 & LMQ & & & 61 & 0 \\
\hline 2168 & LMQ & & & 214 & 0 \\
\hline 2169 & LMQ & & & 297 & 0 \\
\hline 2170 & LMQ & & & 351 & 0 \\
\hline 2171 & LMQ & & & 150 & 0 \\
\hline 2173 & LMQ & & & 215 & 0 \\
\hline 2174 & LMQ & & & 768 & 0 \\
\hline 2181 & LMQ & & & 90 & 0 \\
\hline 2187 & LMQ & & & 90 & 0 \\
\hline 2192 & LMQ & & & 90 & 0 \\
\hline 2195 & LMQ & & & 90 & 0 \\
\hline 2202 & LMQ & & & 90 & 0 \\
\hline 2203 & LMQ & & & 100 & 0 \\
\hline 2204 & LMQ & & & 110 & 0 \\
\hline 2205 & LMQ & & & 958 & 0 \\
\hline 2206 & LMQ & & & 194 & 0 \\
\hline 2315 & QBL & 44.7 & 7.5 & 595 & 0 \\
\hline 2353 & QML & 50.0 & 23.7 & 147 & 0 \\
\hline 2357 & QBL & 50.0 & 7.8 & 240 & 0 \\
\hline 2359 & QBL & 44.4 & 4.2 & 306 & 0 \\
\hline 2416 & LCQ & & & 0 & 0 \\
\hline 2430 & $\mathrm{LCQ}$ & & & 0 & 0 \\
\hline 2445 & $\mathrm{LCQ}$ & & & 0 & 0 \\
\hline 2456 & LCD & & & 0 & 0 \\
\hline 2468 & LCD & & & 0 & 0 \\
\hline 2480 & $\mathrm{LCQ}$ & & & 0 & 0 \\
\hline 2482 & LCD & & & 0 & 0 \\
\hline 2483 & LCQ & & & 0 & 0 \\
\hline 2492 & QBL & 25.5 & 86.2 & 196 & 0 \\
\hline 2505 & LCQ & & & 0 & 0 \\
\hline 2512 & QBL & 46.0 & 77.4 & 100 & 0 \\
\hline 2519 & LCD & & & 0 & 0 \\
\hline 2540 & LCQ & & & 0 & 0 \\
\hline 2546 & $\mathrm{CCQ}$ & 0.0 & 0.7 & 0 & 0 \\
\hline 2590 & $\mathrm{LCQ}$ & & & 0 & 0 \\
\hline 2626 & LCD & & & 0 & 0 \\
\hline 2635 & LCQ & & & 0 & 0 \\
\hline 2650 & LCQ & & & 0 & 0 \\
\hline 2658 & $\mathrm{LCQ}$ & & & 0 & 0 \\
\hline 2676 & LCD & & & 0 & 0 \\
\hline 2693 & $\mathrm{LCQ}$ & & & 0 & 0 \\
\hline 2696 & $\mathrm{QCQ}$ & 1.4 & 2.5 & 0 & 0 \\
\hline 2698 & LCQ & & & 0 & 0 \\
\hline 2702 & $\mathrm{QML}$ & 4.6 & 1.2 & 259 & 0 \\
\hline 2703 & LCQ & & & 0 & 0 \\
\hline 2707 & LCQ & & & 0 & 0 \\
\hline 2708 & LMQ & & & 108 & 0 \\
\hline 2712 & QCL & 50.0 & 100.0 & 0 & 0 \\
\hline 2714 & LCQ & & & 0 & 0 \\
\hline 2733 & QBL & 25.9 & 89.2 & 324 & 0 \\
\hline 2738 & $\mathrm{LCQ}$ & & & 0 & 0 \\
\hline 2758 & LCQ & & & 0 & 0 \\
\hline 2761 & QCL & 50.0 & 100.0 & 0 & 0 \\
\hline 2784 & LCD & & & 0 & 0 \\
\hline 2819 & LCQ & & & 0 & 0 \\
\hline 2823 & LCQ & & & 0 & 0 \\
\hline 2834 & $\mathrm{LCQ}$ & & & 0 & 0 \\
\hline 2862 & LCD & & & 0 & 0 \\
\hline 2880 & QBL & 48.8 & 90.3 & 625 & 0 \\
\hline 2881 & LCQ & & & 0 & 0 \\
\hline 2882 & LMQ & & & 56 & 0 \\
\hline 2894 & $\mathrm{LCQ}$ & & & 0 & 0 \\
\hline 2935 & LMQ & & & 72 & 0 \\
\hline 2957 & QBL & 23.1 & 60.3 & 484 & 0 \\
\hline 2958 & LMQ & & & 42 & 0 \\
\hline 2967 & $\mathrm{QCC}$ & 47.4 & 5.0 & 0 & 0 \\
\hline 2981 & CCQ & 0.0 & 0.7 & 0 & 0 \\
\hline 2987 & $\mathrm{LCQ}$ & & & 0 & 0 \\
\hline 2993 & $\mathrm{LCQ}$ & & & 0 & 0 \\
\hline 3029 & LCD & & & 0 & 0 \\
\hline 3034 & $\mathrm{LCQ}$ & & & 0 & 0 \\
\hline 3049 & $\mathrm{QCQ}$ & 0.8 & 2.5 & 0 & 0 \\
\hline 3060 & QML & 0.2 & 6.2 & 48 & 0 \\
\hline 3080 & $\mathrm{CCQ}$ & 0.0 & 0.7 & 0 & 0 \\
\hline 3083 & $\mathrm{LCQ}$ & & & 0 & 0 \\
\hline 3088 & $\mathrm{LCD}$ & & & 0 & 0 \\
\hline
\end{tabular}


Table 7: Features of QPLIB instances (continued).

\begin{tabular}{|c|c|c|c|c|c|}
\hline & & $Q$ & & & Jariab \\
\hline name & type & $\%$ p.e. & $\% \mathrm{~d}$. & \# b. & \# i. \\
\hline 3089 & $\mathrm{LCQ}$ & & & 0 & 0 \\
\hline 3105 & LCD & & & 0 & 0 \\
\hline 3120 & $\mathrm{LCQ}$ & & & 0 & 0 \\
\hline 3122 & QML & 2.8 & 0.1 & 17136 & 0 \\
\hline 3147 & $\mathrm{LCQ}$ & & & 0 & 0 \\
\hline 3170 & $\mathrm{LCQ}$ & & & 0 & 0 \\
\hline 3177 & $\mathrm{LCQ}$ & & & 0 & 0 \\
\hline 3181 & LMQ & & & 84 & 0 \\
\hline 3185 & LCD & & & 0 & 0 \\
\hline 3192 & LCQ & & & 0 & 0 \\
\hline 3225 & $\mathrm{LCQ}$ & & & 0 & 0 \\
\hline 3240 & LCQ & & & 0 & 0 \\
\hline 3247 & LCQ & & & 0 & 0 \\
\hline 3279 & LMQ & & & 56 & 0 \\
\hline 3297 & $\mathrm{CCQ}$ & 0.0 & 0.7 & 0 & 0 \\
\hline 3307 & QBL & 19.9 & 61.5 & 256 & 0 \\
\hline 3312 & LCD & & & 0 & 0 \\
\hline 3318 & LCQ & & & 0 & 0 \\
\hline 3326 & $\mathrm{QCQ}$ & 2.9 & 2.5 & 0 & 0 \\
\hline 3334 & LCQ & & & 0 & 0 \\
\hline 3337 & $\mathrm{LCQ}$ & & & 0 & 0 \\
\hline 3338 & $\mathrm{LCQ}$ & & & 0 & 0 \\
\hline 3347 & $\mathrm{QBL}$ & 51.8 & 85.8 & 676 & 0 \\
\hline 3358 & LCQ & & & 0 & 0 \\
\hline 3361 & QBL & 28.3 & 35.5 & 1024 & 0 \\
\hline 3369 & LCQ & & & 0 & 0 \\
\hline 3380 & QBL & 3.4 & 0.1 & 8904 & 0 \\
\hline 3385 & LCQ & & & 0 & 0 \\
\hline 3387 & $\mathrm{LCQ}$ & & & 0 & 0 \\
\hline 3402 & QBL & 47.2 & 81.5 & 144 & 0 \\
\hline 3413 & QBL & 45.0 & 9.0 & 400 & 0 \\
\hline 3416 & LCQ & & & 0 & 0 \\
\hline 3496 & LGQ & & & 200 & 56 \\
\hline 3502 & LMQ & & & 10920 & 0 \\
\hline 3505 & LMQ & & & 201 & 0 \\
\hline 3506 & QBN & 48.4 & 0.8 & 496 & 0 \\
\hline 3508 & LMQ & & & 2450 & 0 \\
\hline 3510 & LMQ & & & 105 & 0 \\
\hline 3511 & LMQ & & & 2450 & 0 \\
\hline 3512 & LMQ & & & 72 & 0 \\
\hline 3513 & LMQ & & & 123 & 0 \\
\hline 3514 & LMQ & & & 15 & 0 \\
\hline 3515 & LMQ & & & 352 & 0 \\
\hline 3522 & LMQ & & & 42 & 0 \\
\hline 3523 & QML & 50.0 & 13.2 & 155 & 0 \\
\hline 3524 & LMQ & & & 132 & 0 \\
\hline 3525 & QGQ & 47.5 & 0.1 & 0 & 1662 \\
\hline 3529 & LMQ & & & 38 & 0 \\
\hline 3533 & LMQ & & & 240 & 0 \\
\hline 3547 & DML & 0.0 & 16.7 & 462 & 0 \\
\hline 3549 & LMQ & & & 650 & 0 \\
\hline 3554 & QML & 12.0 & 100.0 & 14 & 0 \\
\hline 3562 & LIQ & & & 7 & 56 \\
\hline 3565 & $\mathrm{QBN}$ & 47.8 & 1.4 & 276 & 0 \\
\hline 3580 & LMQ & & & 108 & 0 \\
\hline 3582 & LMQ & & & 184 & 0 \\
\hline 3584 & QBL & 43.9 & 8.0 & 528 & 0 \\
\hline 3587 & QBL & 50.0 & 12.7 & 240 & 0 \\
\hline 3588 & LMQ & & & 600 & 0 \\
\hline 3592 & $\mathrm{QML}$ & 50.0 & 0.2 & 225 & 0 \\
\hline 3596 & LMQ & & & 104 & 0 \\
\hline 3600 & LMQ & & & 112 & 0 \\
\hline 3605 & LMQ & & & 160 & 0 \\
\hline 3614 & QBL & 50.0 & 12.7 & 210 & 0 \\
\hline 3620 & LMQ & & & 187 & 0 \\
\hline 3621 & LMQ & & & 109 & 0 \\
\hline 3622 & LMQ & & & 25 & 0 \\
\hline 3624 & LMQ & & & 40 & 0 \\
\hline 3625 & $\mathrm{LMQ}$ & & & 46 & 0 \\
\hline 3631 & LMQ & & & 750 & 0 \\
\hline 3642 & $\mathrm{QBN}$ & 48.9 & 0.4 & 1035 & 0 \\
\hline 3643 & LGQ & & & 216 & 72 \\
\hline 3645 & LMQ & & & 101 & 0 \\
\hline 3646 & LMQ & & & 20 & 0 \\
\hline 3648 & LMQ & & & 40 & 0 \\
\hline 3650 & $\mathrm{QBN}$ & 48.8 & 0.4 & 946 & 0 \\
\hline 3651 & LMQ & & & 137 & 0 \\
\hline 3659 & LGQ & & & 0 & 960 \\
\hline 3661 & LMQ & & & 10816 & 0 \\
\hline 3662 & LMQ & & & 144 & 0 \\
\hline 3670 & LMQ & & & 54 & 0 \\
\hline 3676 & LMQ & & & 30 & 0 \\
\hline 3677 & LMQ & & & 30 & 0 \\
\hline 3678 & LMD & & & 200 & 0 \\
\hline 3680 & LMQ & & & 92 & 0 \\
\hline 3683 & LMQ & & & 126 & 0 \\
\hline 3690 & LMQ & & & 20 & 0 \\
\hline 3692 & LMQ & & & 128 & 0 \\
\hline 3693 & QBN & 48.9 & 0.3 & 1128 & 0 \\
\hline 3694 & DML & 0.0 & 0.1 & 40 & 0 \\
\hline 3697 & LMQ & & & 168 & 0 \\
\hline
\end{tabular}


Table 7: Features of QPLIB instances (continued).

\begin{tabular}{|c|c|c|c|c|c|}
\hline & & & & & ariabl \\
\hline name & type & $\%$ p.e. & $\% \mathrm{~d}$ & \# b. & \# i. \\
\hline 3698 & DML & 0.0 & 0.1 & 30 & 0 \\
\hline 3699 & LMQ & & & 116 & 0 \\
\hline 3701 & LMQ & & & 60 & 0 \\
\hline 3703 & QBL & 46.7 & 84.6 & 225 & 0 \\
\hline 3705 & QBN & 48.1 & 1.0 & 378 & 0 \\
\hline 3706 & QBN & 48.6 & 0.6 & 703 & 0 \\
\hline 3708 & DML & 0.0 & 0.1 & 14 & 0 \\
\hline 3709 & QBL & 48.0 & 91.8 & 600 & 0 \\
\hline 3713 & LMQ & & & 42 & 0 \\
\hline 3714 & QBL & 97.5 & 32.5 & 120 & 0 \\
\hline 3719 & LMQ & & & 133 & 0 \\
\hline 3725 & LMQ & & & 81 & 0 \\
\hline 3726 & LMQ & & & 116 & 0 \\
\hline 3727 & LMQ & & & 20 & 0 \\
\hline 3728 & LMQ & & & 72 & 0 \\
\hline 3729 & LMQ & & & 650 & 0 \\
\hline 3733 & LMQ & & & 46 & 0 \\
\hline 3734 & LMQ & & & 38 & 0 \\
\hline 3738 & QBN & 48.3 & 0.9 & 435 & 0 \\
\hline 3745 & QBN & 48.0 & 1.2 & 325 & 0 \\
\hline 3748 & LMQ & & & 75 & 0 \\
\hline 3750 & QBL & 98.6 & 32.9 & 210 & 0 \\
\hline 3751 & QBL & 98.0 & 32.7 & 150 & 0 \\
\hline 3752 & QBL & 45.5 & 4.1 & 462 & 0 \\
\hline 3757 & QBL & 34.4 & 1.7 & 552 & 0 \\
\hline 3762 & QBL & 50.0 & 28.0 & 90 & 0 \\
\hline 3772 & QBL & 50.0 & 3.8 & 380 & 0 \\
\hline 3775 & QBL & 98.3 & 32.8 & 180 & 0 \\
\hline 3780 & LIQ & & & 12 & 156 \\
\hline 3785 & LMQ & & & 200 & 0 \\
\hline 3790 & QML & 9.7 & 100.0 & 7 & 0 \\
\hline 3792 & DML & 0.0 & 0.1 & 20 & 0 \\
\hline 3794 & LMQ & & & 576 & 0 \\
\hline 3797 & LMQ & & & 48 & 0 \\
\hline 3798 & LMQ & & & 54 & 0 \\
\hline 3803 & QBL & 42.6 & 14.1 & 190 & 0 \\
\hline 3809 & LMQ & & & 224 & 0 \\
\hline 3813 & LMQ & & & 15 & 0 \\
\hline 3814 & QMQ & 4.2 & 16.0 & 2 & 0 \\
\hline 3815 & QBL & 50.0 & 3.1 & 192 & 0 \\
\hline 3816 & LMQ & & & 70 & 0 \\
\hline 3822 & QBN & 48.8 & 0.5 & 861 & 0 \\
\hline 3825 & LMQ & & & 60 & 0 \\
\hline 3832 & QBN & 48.5 & 0.7 & 561 & 0 \\
\hline 3834 & QBL & 60.0 & 98.0 & 50 & 0 \\
\hline 3838 & QBN & 48.7 & 0.5 & 780 & 0 \\
\hline 3840 & LMQ & & & 2401 & 0 \\
\hline 3841 & QBL & 44.0 & 10.2 & 300 & 0 \\
\hline 3850 & QBN & 49.0 & 0.3 & 1225 & 0 \\
\hline 3852 & QBN & 47.6 & 1.6 & 231 & 0 \\
\hline 3854 & LMQ & & & 40 & 0 \\
\hline 3855 & LMQ & & & 400 & 0 \\
\hline 3856 & LMQ & & & 168 & 0 \\
\hline 3857 & LMQ & & & 201 & 0 \\
\hline 3859 & LMQ & & & 600 & 0 \\
\hline 3860 & QBL & 44.8 & 8.7 & 435 & 0 \\
\hline 3861 & DML & 0.0 & 0.1 & 30 & 0 \\
\hline 3863 & LMQ & & & 625 & 0 \\
\hline 3865 & QBL & 48.0 & 90.7 & 525 & 0 \\
\hline 3870 & QML & 42.9 & 23.4 & 116 & 0 \\
\hline 3871 & DML & 0.0 & 0.1 & 25 & 0 \\
\hline 3872 & LMQ & & & 95 & 0 \\
\hline 3877 & QBN & 48.6 & 0.6 & 630 & 0 \\
\hline 3879 & LMQ & & & 10920 & 0 \\
\hline 3883 & $\mathrm{QBL}$ & 50.0 & 17.8 & 182 & 0 \\
\hline 3913 & CBL & 0.0 & 100.0 & 300 & 0 \\
\hline 3923 & QBL & 53.7 & 8.0 & 395 & 0 \\
\hline 3931 & QBL & 50.3 & 8.0 & 316 & 0 \\
\hline 3980 & CBL & 0.0 & 100.0 & 235 & 0 \\
\hline 4095 & CMQ & 0.0 & 100.0 & 400 & 0 \\
\hline 4270 & $\mathrm{CML}$ & 0.0 & 25.1 & 400 & 0 \\
\hline 4455 & LMQ & & & 3000 & 0 \\
\hline 4722 & LMQ & & & 2000 & 0 \\
\hline 4805 & LMQ & & & 2000 & 0 \\
\hline 5023 & LMQ & & & 3000 & 0 \\
\hline 5442 & LMQ & & & 2000 & 0 \\
\hline 5527 & DML & 0.0 & 0.1 & 4492 & 0 \\
\hline 5543 & DML & 0.0 & 0.1 & 4514 & 0 \\
\hline 5554 & LMQ & & & 4492 & 0 \\
\hline 5573 & LMQ & & & 4450 & 0 \\
\hline 5577 & DML & 0.0 & 0.1 & 1118 & 0 \\
\hline 5721 & QBN & 49.0 & 76.8 & 300 & 0 \\
\hline 5725 & QBN & 50.1 & 1.7 & 343 & 0 \\
\hline 5755 & QBN & 50.0 & 1.0 & 400 & 0 \\
\hline 5875 & QBN & 50.0 & 78.9 & 200 & 0 \\
\hline 5881 & QBN & 49.2 & 29.5 & 120 & 0 \\
\hline 5882 & QBN & 49.3 & 78.1 & 150 & 0 \\
\hline 5909 & $\mathrm{QBN}$ & 50.0 & 9.6 & 250 & 0 \\
\hline 5922 & QBN & 49.8 & 9.8 & 500 & 0 \\
\hline 5924 & DML & 0.0 & 0.7 & 300 & 0 \\
\hline 5925 & LMQ & & & 100 & 0 \\
\hline
\end{tabular}


Table 7: Features of QPLIB instances (continued).

\begin{tabular}{|c|c|c|c|c|c|}
\hline & & & & & Variabl \\
\hline name & type & \% p.e. & $\% \mathrm{~d}$ & \# b. & \# i. \\
\hline 5926 & LMQ & & & 2400 & 0 \\
\hline 5927 & LMQ & & & 2400 & 0 \\
\hline 5935 & QBL & 49.0 & 99.0 & 100 & 0 \\
\hline 5944 & QBL & 49.0 & 99.0 & 100 & 0 \\
\hline 5962 & QBL & 49.3 & 99.3 & 150 & 0 \\
\hline 5971 & QBL & 49.3 & 99.3 & 150 & 0 \\
\hline 5980 & QBL & 49.3 & 99.3 & 150 & 0 \\
\hline 6287 & LCQ & & & 0 & 0 \\
\hline 6310 & LCQ & & & 0 & 0 \\
\hline 6311 & LCQ & & & 0 & 0 \\
\hline 6324 & QBL & 50.6 & 31.3 & 640 & 0 \\
\hline 6487 & QBL & 35.0 & 20.9 & 618 & 0 \\
\hline 6597 & QBL & 45.7 & 97.3 & 600 & 0 \\
\hline 6647 & QBL & 70.0 & 7.2 & 627 & 0 \\
\hline 6757 & QBL & 18.5 & 4.7 & 2046 & 0 \\
\hline 6764 & QBL & 19.1 & 4.7 & 2071 & 0 \\
\hline 6799 & QBL & 18.7 & 4.7 & 2075 & 0 \\
\hline 6941 & QBL & 18.7 & 4.5 & 2203 & 0 \\
\hline 7127 & QBL & 50.6 & 6.8 & 1000 & 0 \\
\hline 7139 & QBL & 53.3 & 89.2 & 180 & 0 \\
\hline 7144 & QBL & 53.2 & 89.6 & 220 & 0 \\
\hline 7149 & QBL & 53.0 & 89.6 & 264 & 0 \\
\hline 7154 & QBL & 52.9 & 89.7 & 312 & 0 \\
\hline 7159 & QBL & 52.5 & 89.7 & 364 & 0 \\
\hline 7164 & QBL & 52.4 & 89.7 & 420 & 0 \\
\hline 7579 & LMD & & & 100 & 0 \\
\hline 8009 & LMQ & & & 101 & 0 \\
\hline 8153 & LMQ & & & 31 & 0 \\
\hline 8381 & LMQ & & & 51 & 0 \\
\hline 8495 & DCL & 0.0 & 0.1 & 0 & 0 \\
\hline 8500 & DCL & 0.0 & 0.1 & 0 & 0 \\
\hline 8505 & QCL & 49.9 & 0.1 & 0 & 0 \\
\hline 8515 & CCL & 0.0 & 0.1 & 0 & 0 \\
\hline 8547 & DCL & 0.0 & 0.1 & 0 & 0 \\
\hline 8553 & QCQ & 0.0 & 0.1 & 0 & 0 \\
\hline 8559 & CCL & 0.0 & 0.1 & 0 & 0 \\
\hline 8567 & CCL & 0.0 & 0.1 & 0 & 0 \\
\hline 8585 & $\mathrm{DCQ}$ & 0.0 & 0.1 & 0 & 0 \\
\hline 8595 & $\mathrm{DCQ}$ & 0.0 & 0.1 & 0 & 0 \\
\hline 8602 & DCL & 0.0 & 0.1 & 0 & 0 \\
\hline 8605 & DCQ & 0.0 & 0.1 & 0 & 0 \\
\hline 8616 & DCL & 0.0 & 0.1 & 0 & 0 \\
\hline 8683 & DCQ & 0.0 & 0.1 & 0 & 0 \\
\hline 8685 & $\mathrm{DCQ}$ & 0.0 & 0.1 & 0 & 0 \\
\hline 8758 & $\mathrm{QCQ}$ & 4.3 & 50.0 & 0 & 0 \\
\hline 8777 & QCL & 34.6 & 0.1 & 0 & 0 \\
\hline 8784 & QCC & 49.5 & 1.0 & 0 & 0 \\
\hline 8785 & DCL & 0.0 & 0.1 & 0 & 0 \\
\hline 8790 & $\mathrm{CCB}$ & 0.0 & 0.1 & 0 & 0 \\
\hline 8792 & $\mathrm{CCB}$ & 0.0 & 0.1 & 0 & 0 \\
\hline 8803 & DCQ & 0.0 & 0.1 & 0 & 0 \\
\hline 8810 & $\mathrm{DCQ}$ & 0.0 & 0.1 & 0 & 0 \\
\hline 8815 & QCD & 0.1 & 25.0 & 0 & 0 \\
\hline 8845 & CCL & 0.0 & 59.8 & 0 & 0 \\
\hline 8906 & CCL & 0.0 & 3.0 & 0 & 0 \\
\hline 8938 & DCL & 0.0 & 0.1 & 0 & 0 \\
\hline 8991 & $\mathrm{CCB}$ & 0.0 & 0.1 & 0 & 0 \\
\hline 9002 & DCL & 0.0 & 0.1 & 0 & 0 \\
\hline 9004 & QCQ & 25.0 & 0.1 & 0 & 0 \\
\hline 9008 & DCL & 0.0 & 0.1 & 0 & 0 \\
\hline 9030 & QIL & 0.1 & 0.1 & 0 & 10000 \\
\hline 9048 & QIL & 29.7 & 18.2 & 0 & 202 \\
\hline 10001 & LMC & & & 426 & 0 \\
\hline 10002 & LMC & & & 426 & 0 \\
\hline 10003 & LMC & & & 999 & 0 \\
\hline 10004 & LMC & & & 150 & 0 \\
\hline 10005 & LMC & & & 1000 & 0 \\
\hline 10006 & LMC & & & 1875 & 0 \\
\hline 10007 & LMC & & & 2625 & 0 \\
\hline 10008 & LMC & & & 713 & 0 \\
\hline 10009 & LMC & & & 473 & 0 \\
\hline 10010 & LMC & & & 262 & 0 \\
\hline 10011 & LMC & & & 1258 & 0 \\
\hline 10012 & LMC & & & 835 & 0 \\
\hline 10013 & LMQ & & & 3600 & 0 \\
\hline 10014 & LMQ & & & 3600 & 0 \\
\hline 10015 & LMQ & & & 3600 & 0 \\
\hline 10016 & LMQ & & & 3600 & 0 \\
\hline 10017 & LMQ & & & 4800 & 0 \\
\hline 10018 & LMQ & & & 4800 & 0 \\
\hline 10019 & LMQ & & & 4800 & 0 \\
\hline 10020 & LMQ & & & 4800 & 0 \\
\hline 10021 & LMQ & & & 3000 & 0 \\
\hline 10022 & LMQ & & & 3000 & 0 \\
\hline 10023 & LMQ & & & 3000 & 0 \\
\hline 10024 & LMQ & & & 3000 & 0 \\
\hline 10025 & CMQ & 0.0 & 100.0 & 400 & 0 \\
\hline 10026 & CMQ & 0.0 & 100.0 & 400 & 0 \\
\hline 10027 & CMQ & 0.0 & 100.0 & 400 & 0 \\
\hline 10028 & CMQ & 0.0 & 100.0 & 400 & 0 \\
\hline 10029 & CMQ & 0.0 & 00.0 & 400 & 0 \\
\hline
\end{tabular}


Table 7: Features of QPLIB instances (continued).

\begin{tabular}{|c|c|c|c|c|c|c|c|c|c|c|}
\hline \multirow[b]{2}{*}{ name } & \multirow[b]{2}{*}{ type } & \multicolumn{2}{|c|}{$Q^{0}$} & \multicolumn{3}{|c|}{ Variables } & \multicolumn{4}{|c|}{ Constraints } \\
\hline & & $\%$ p.e. & $\%$ d. & \# b. & \# i. & \# c. & \# 1 & \# q. & \# c. & \# v. \\
\hline 10030 & LMQ & & & 3000 & 0 & 12000 & 9001 & 3000 & 0 & 3000 \\
\hline 10031 & LMQ & & & 3000 & 0 & 12000 & 9001 & 3000 & 0 & 3000 \\
\hline 10032 & LMQ & & & 3000 & 0 & 12000 & 9001 & 3000 & 0 & 3000 \\
\hline 10033 & LMQ & & & 3000 & 0 & 12000 & 9001 & 3000 & 0 & 3000 \\
\hline 10034 & $\mathrm{DCL}$ & 0.0 & 0.2 & 0 & 0 & 40400 & 40200 & 0 & 0 & 802 \\
\hline 10035 & LCQ & & & 0 & 0 & 40401 & 40000 & 200 & 1 & 1200 \\
\hline 10036 & $\mathrm{LCQ}$ & & & 0 & 0 & 40401 & 40000 & 200 & 1 & 1200 \\
\hline 10037 & $\mathrm{LCQ}$ & & & 0 & 0 & 40401 & 200 & 40000 & 1 & 400 \\
\hline 10038 & DCL & 0.0 & 0.1 & 0 & 0 & 160800 & 160400 & 0 & 0 & 1602 \\
\hline 10039 & LCQ & & & 0 & 0 & 12097 & 11713 & 193 & 0 & 384 \\
\hline 10040 & QBL & 8.8 & 92.6 & 125 & 0 & 0 & 6 & 0 & 0 & 0 \\
\hline 10041 & QBL & 4.0 & 99.9 & 125 & 0 & 0 & 6 & 0 & 0 & 0 \\
\hline 10042 & QBL & 0.8 & 99.9 & 125 & 0 & 0 & 5 & 0 & 0 & 0 \\
\hline 10043 & QBL & 4.7 & 96.7 & 150 & 0 & 0 & 10 & 0 & 0 & 0 \\
\hline 10044 & QBL & 8.0 & 97.0 & 150 & 0 & 0 & 6 & 0 & 0 & 0 \\
\hline 10045 & QBL & 8.7 & 99.4 & 150 & 0 & 0 & 10 & 0 & 0 & 0 \\
\hline 10046 & QBL & 0.7 & 92.1 & 150 & 0 & 0 & 6 & 0 & 0 & 0 \\
\hline 10047 & QBL & 4.7 & 99.9 & 150 & 0 & 0 & 10 & 0 & 0 & 0 \\
\hline 10048 & QBL & 1.3 & 99.9 & 150 & 0 & 0 & 5 & 0 & 0 & 0 \\
\hline 10049 & QBL & 2.7 & 99.9 & 150 & 0 & 0 & 10 & 0 & 0 & 0 \\
\hline 10050 & CBL & 0.0 & 100.0 & 150 & 0 & 0 & 5 & 0 & 0 & 0 \\
\hline 10051 & QBL & 2.0 & 99.9 & 150 & 0 & 0 & 10 & 0 & 0 & 0 \\
\hline 10052 & QBL & 1.3 & 99.9 & 150 & 0 & 0 & 6 & 0 & 0 & 0 \\
\hline 10053 & QBL & 0.7 & 99.9 & 150 & 0 & 0 & 10 & 0 & 0 & 0 \\
\hline 10054 & QBL & 4.6 & 90.1 & 175 & 0 & 0 & 11 & 0 & 0 & 0 \\
\hline 10055 & QBL & 2.9 & 91.5 & 175 & 0 & 0 & 5 & 0 & 0 & 0 \\
\hline 10056 & CBL & 0.0 & 98.8 & 175 & 0 & 0 & 5 & 0 & 0 & 0 \\
\hline 10057 & QBL & 9.5 & 80.5 & 200 & 0 & 0 & 11 & 0 & 0 & 0 \\
\hline 10058 & QBL & 7.5 & 88.0 & 200 & 0 & 0 & 11 & 0 & 0 & 0 \\
\hline 10059 & QBL & 18.5 & 97.3 & 200 & 0 & 0 & 10 & 0 & 0 & 0 \\
\hline 10060 & QBL & 8.0 & 91.5 & 200 & 0 & 0 & 10 & 0 & 0 & 0 \\
\hline 10061 & QBL & 9.0 & 97.6 & 200 & 0 & 0 & 5 & 0 & 0 & 0 \\
\hline 10062 & QBL & 9.5 & 97.0 & 200 & 0 & 0 & 10 & 0 & 0 & 0 \\
\hline 10063 & QBL & 3.0 & 99.5 & 200 & 0 & 0 & 5 & 0 & 0 & 0 \\
\hline 10064 & QBL & 2.0 & 99.8 & 200 & 0 & 0 & 11 & 0 & 0 & 0 \\
\hline 10065 & QBL & 1.0 & 99.0 & 200 & 0 & 0 & 11 & 0 & 0 & 0 \\
\hline 10066 & QBL & 1.5 & 100.0 & 200 & 0 & 0 & 11 & 0 & 0 & 0 \\
\hline 10067 & QBL & 2.5 & 99.7 & 200 & 0 & 0 & 5 & 0 & 0 & 0 \\
\hline 10068 & QBL & 2.0 & 99.9 & 200 & 0 & 0 & 11 & 0 & 0 & 0 \\
\hline 10069 & CBL & 0.0 & 96.8 & 200 & 0 & 0 & 10 & 0 & 0 & 0 \\
\hline 10070 & QBL & 1.5 & 99.9 & 200 & 0 & 0 & 11 & 0 & 0 & 0 \\
\hline 10071 & QBL & 1.0 & 99.0 & 200 & 0 & 0 & 11 & 0 & 0 & 0 \\
\hline 10072 & QBL & 12.0 & 90.1 & 75 & 0 & 0 & 10 & 0 & 0 & 0 \\
\hline 10073 & QBL & 10.7 & 84.9 & 75 & 0 & 0 & 6 & 0 & 0 & 0 \\
\hline 10074 & QBL & 1.3 & 100.0 & 75 & 0 & 0 & 10 & 0 & 0 & 0 \\
\hline
\end{tabular}

\section{B. The File Format}

The QPLIB format is defined in Table 8, with the notation of $\S 2$.

The data is in free format (blanks separate values), but must occur in the order given here. Any blank lines, or lines starting with any of the characters !, \% or \# are ignored. Each term in the first column of Table 8 denotes a required value. Any strings beyond those required on a given line will be regarded as comments and ignored. Real values may either by in decimal or exponential formats; for the latter, the exponent may be preceded by either the character D or E, e.g. $12.56 \mathrm{D}+2$ or $12.56 \mathrm{E}+2$. Variable indices, $j$, must lie in the range $1 \leq j \leq n$, while constraint indices, $i$, must satisfy $1 \leq i \leq m$, that is they are both one-based. The case for character strings is irrelevant.

Table 8: The QPLIB file format: refer to the notes after the table for more details.

\begin{tabular}{|llr|}
\hline data & description & note \\
\hline name & problem name (character string) & \\
type & problem type (character string) & {$[1]$} \\
sense & one of the words minimize or maximize (character string) & \\
\hline$n$ & number of variables (integer) & \\
\hline
\end{tabular}


Table 8: The QPLIB file format (continued)

\begin{tabular}{|c|c|c|}
\hline data & description & note \\
\hline$m$ & number of constraints (integer) & [2] \\
\hline$n^{Q^{0}}$ & number of nonzeros (integer) in lower triangle of $Q^{0}$ & [3] \\
\hline$h \quad k \quad Q_{h k}^{0}$ & $\begin{array}{l}\text { row and column indices (integers) and value (real) for each } \\
\text { nonzero entry of } Q^{0} \text {, if } n^{Q^{0}}>0 \text {, one triple on each line }\end{array}$ & [3] \\
\hline$b_{d}^{0}$ & default value (real) for entries in $b^{0}$ & \\
\hline & number of non-default entries (integer) in $b^{0}$ & \\
\hline$j b_{j}^{0}$ & $\begin{array}{l}\text { index (integer) and value (real) for each non-default term in } b^{0} \\
\text { if } n^{b^{0}}>0 \text {, one pair per line }\end{array}$ & \\
\hline$q^{0}$ & constant part of the objective function & \\
\hline$\sum_{i \in \mathcal{M}} n^{Q^{i}}$ & $\begin{array}{l}\text { number of nonzeros (integer) in lower triangles of } Q^{i} \text {, summed } \\
\text { over all } i \in \mathcal{M}\end{array}$ & {$[2,4]$} \\
\hline$i h k Q_{h k}^{i}$ & $\begin{array}{l}i \text {, row and column indices (integers) and value (real) for each } \\
\text { entry of } Q^{i} \text { for every } i \in \mathcal{M} \text {, if } n^{Q^{i}}>0 \text {, one quadruple on each } \\
\text { line }\end{array}$ & \\
\hline$\sum n^{b^{i}}$ & number of nonzeros (integer) in $b^{i}$, summed over all $i \in \mathcal{M}$ & [2] \\
\hline$i_{i \in \mathcal{M}}^{i} b_{j}^{i}$ & $\begin{array}{l}i \text { and index (integers) and value (real) for each nonzero entry } \\
\text { of } b^{i} \text { for every } i \in \mathcal{M} \text {, if } n^{b^{i}}>0 \text {, one triple on each line }\end{array}$ & [2] \\
\hline$c_{\infty}$ & $\begin{array}{l}\text { value (real) for infinity for constraint or variable bounds-any } \\
\text { bound greater than or equal to this in, absolute value, is infinite }\end{array}$ & \\
\hline$c_{l, d}$ & default value (real) for entries in $c_{l}$ & [2] \\
\hline$n^{c_{l, d}}$ & number of non-default entries (integ & {$[2]$} \\
\hline$i c_{l}^{i}$ & $\begin{array}{l}\text { index (integer) and value (real) for each non-default term in } \\
c_{l, d} \text {, if } n^{c_{l, d}}>0 \text {, one pair per line }\end{array}$ & [2] \\
\hline$c_{u, d}$ & default value (real) for entries in $c_{u}$ & [2] \\
\hline$n^{c_{u, d}}$ & number of non-default entries (integer) in $c_{u}$ & [2] \\
\hline$i c_{u}^{i}$ & $\begin{array}{l}\text { index (integer) and value (real) for each non-default term in } \\
c_{u, d} \text {, if } n^{c_{u, d}}>0 \text {, one pair per line }\end{array}$ & [2] \\
\hline$l_{d}$ & default value (real) for entries in $l$ & [6] \\
\hline$n^{l_{d}}$ & number of non-default entries (integer) in $l$ & [6] \\
\hline$i l_{i}$ & $\begin{array}{l}\text { index (integer) and value (real) for each non-default term in } l \text {, } \\
\text { if } n^{l_{d}}>0 \text {, one pair per line }\end{array}$ & [6] \\
\hline$u_{d}$ & default value (real) for entries in $u$ & [6] \\
\hline$n^{u_{d}}$ & number of non-default entries (integer) in $u$ & [6] \\
\hline$i u_{i}$ & $\begin{array}{l}\text { index (integer) and value (real) for each non-default term in } u \text {, } \\
\text { if } n^{u_{d}}>0 \text {, one pair per line }\end{array}$ & [6] \\
\hline$v_{d}$ & $\begin{array}{l}\text { default variable type (integer, } 0 \text { for continuous variables, } 1 \text { for } \\
\text { integer variables, } 2 \text { for binary variables) }\end{array}$ & [5] \\
\hline$n^{v}$ & number of non-default variables (integer) & [5] \\
\hline$i v_{i}$ & $\begin{array}{l}\text { index and type (integers) for each non-default variable type, if } \\
n^{v}>0 \text {, one pair per line }\end{array}$ & [5] \\
\hline$x_{d}^{0}$ & $\begin{array}{l}\text { default value (real) for the components of the starting point } x^{0} \\
\text { for the variables } x\end{array}$ & \\
\hline
\end{tabular}


Table 8: The QPLIB file format (continued)

\begin{tabular}{|c|c|c|}
\hline data & description & note \\
\hline$n^{x^{0}}$ & number of non-default starting entries (integer) in $x$ & \\
\hline$i x_{i}^{0}$ & $\begin{array}{l}\text { index (integer) and value (real) for each non-default starting } \\
\text { value in } x^{0} \text {, if } n^{x^{0}}>0 \text {, one pair per line }\end{array}$ & \\
\hline$y_{d}^{0}$ & $\begin{array}{l}\text { default value (real) for the components of the starting point } y^{0} \\
\text { for the Lagrange multipliers } y \text { for the general constraints }\end{array}$ & [2] \\
\hline$n^{y^{0}}$ & number of non-default starting entries (integer) in $y$ & [2] \\
\hline$i y_{i}^{0}$ & $\begin{array}{l}\text { index (integer) and value (real) for each non-default starting } \\
\text { value in } y^{0} \text {, if } n^{y^{0}}>0 \text {, one pair per line }\end{array}$ & {$[2]$} \\
\hline$z_{d}^{0}$ & $\begin{array}{l}\text { default value (real) for the components of the starting point } z^{0} \\
\text { for the dual variables } z \text { for the simple bound constraints }\end{array}$ & \\
\hline$n^{z^{0}}$ & number of non-default starting entries (integer) in $z$ & \\
\hline$i z_{i}^{0}$ & $\begin{array}{l}\text { index (integer) and value (real) for each non-default starting } \\
\text { value in } z^{0} \text {, if } n^{z^{0}}>0 \text {, one pair per line }\end{array}$ & \\
\hline$\overline{n_{d}^{x}}$ & $\begin{array}{l}\text { number of non-default names (integer) of variables-default } \\
\text { for variable } i \text { is the character string representing the numerical } \\
\text { value } i\end{array}$ & \\
\hline$j$ var_name ${ }_{j}$ & $\begin{array}{l}\text { index (integer) and name (character string) for each non-default } \\
\text { variable name, if } n_{d}^{x}>0 \text {, one pair per line }\end{array}$ & \\
\hline$n_{d}^{c}$ & $\begin{array}{l}\text { number of non-default names (integer) of general constraints- } \\
\text { default for constraint } i \text { is the character string representing the } \\
\text { numerical value } i\end{array}$ & \\
\hline$i$ cons_name ${ }_{i}$ & $\begin{array}{l}\text { index (integer) and name (character string) for each non-default } \\
\text { constraint name, if } n_{d}^{c}>0 \text {, one pair per line }\end{array}$ & \\
\hline
\end{tabular}

[1] The problem type is represented by a three character string as given in $\S 2.2 .1$

[2] For problems of type ${ }^{* *} N$ or ${ }^{* *} B$, these lines/sections are omitted.

[3] For problems of type $L^{* *}$, this section is omitted.

[4] For problems of type ${ }^{* *} N,{ }^{* *} B$ or ${ }^{* *} L$, this section is omitted.

[5] For problems of type ${ }^{*} C^{*},{ }^{*} B^{*}$ or ${ }^{*} I^{*}$, this section is omitted. For problems of type ${ }^{*} I^{*}$, binary variables should be specified as integer variables with lower and upper bounds 0 and 1 .

[6] For problems of type ${ }^{*} B^{*}$, this section is omitted.

Binary variables defined either implicitly via the type ${ }^{*} B^{*}$ or explicitly in the variable type section will be assumed to have lower and upper bounds 0 and 1 , and this will override any explicit bounds $l_{d}, u_{d}, l_{i}$, and $u_{i}$ set in the lower and upper bound sections. To fix a binary variable to 0 or 1 , its variable type should be changed to continuous or general integer and the corresponding lower and upper bounds set accordingly in the lower and upper bound sections. 
As a simple example, consider the mixed-integer QP

$$
\begin{aligned}
& \min _{x \in \mathbb{R}^{3}} x_{1}^{2}+x_{2}^{2}+x_{3}^{2}-x_{1} x_{2}-x_{2} x_{3}-0.2 x_{1}-0.4 x_{2}-0.2 x_{3} \\
& \text { subject to } 1 \leq x_{1}+x_{2}, 1 \leq x_{1}+x_{3}, 0 \leq x_{1} \leq 1,0 \leq x_{2} \leq 2, \text { and binary } x_{3},
\end{aligned}
$$

for which the Hessian of the objective function is

$$
Q^{0}=\left(\begin{array}{ccc}
2 & -1 & 0 \\
-1 & 2 & -1 \\
0 & -1 & 2
\end{array}\right)
$$

This may then be represented in QPLIB format as follows:

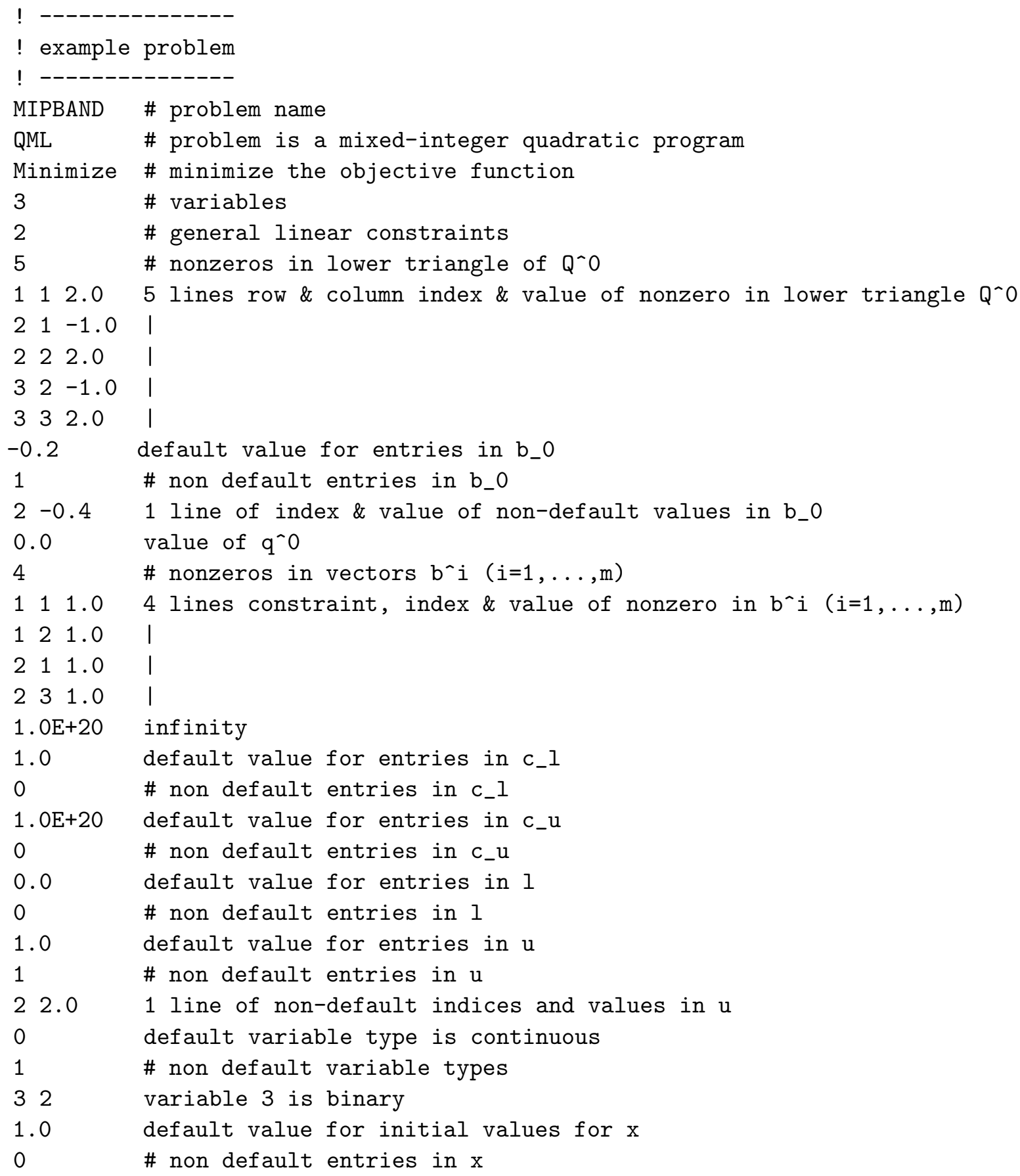


0.0 default value for initial values for $y$

$0 \quad$ \# non default entries in $\mathrm{y}$

0.0 default value for initial values for $z$

$0 \quad$ \# non default entries in $\mathrm{z}$

$0 \quad$ \# non default names for variables

$0 \quad$ \# non default names for constraints 